\title{
Existence and regularity of strict critical subsolutions in the stationary ergodic setting
}

\author{
Andrea Davini *, Antonio Siconolfi \\ Dip. di Matematica, Università di Roma “La Sapienza”, P.le Aldo Moro 2, 00185 Roma, Italy \\ Received 11 July 2013; received in revised form 30 September 2014; accepted 30 September 2014 \\ Available online 16 October 2014
}

\begin{abstract}
We prove that any continuous and convex stationary ergodic Hamiltonian admits critical subsolutions, which are strict outside the random Aubry set. They make up, in addition, a dense subset of all critical subsolutions with respect to a suitable metric. If the Hamiltonian is additionally assumed of Tonelli type, then there exist strict subsolutions of class $C^{1,1}$ in $\mathbb{R}^{N}$. The proofs are based on the use of Lax-Oleinik semigroups and their regularizing properties in the stationary ergodic environment, as well as on a generalized notion of Aubry set.

(c) 2014 L'Association Publications de l'Institut Henri Poincaré. Published by Elsevier B.V. All rights reserved.
\end{abstract}

MSC: 35D40; 35B27; 35F21; 49L25

Keywords: Stationary ergodic setting; Weak KAM Theory; Homogenization; Viscosity solutions

\section{Introduction}

Throughout the paper we deal with a Hamiltonian $H(x, p, \omega)$ defined in $\mathbb{R}^{N} \times \mathbb{R}^{N} \times \Omega$, where $\Omega$ is a probability space which is separable, in a suitable measure-theoretic sense. It is assumed that $\mathbb{R}^{N}$ acts ergodically on $\Omega$ and that $H$ satisfies a stationarity property with respect to such action. As well known, this frame, usually called stationary ergodic, generalizes periodic, quasi-periodic and almost-periodic settings. Besides the basic convexity and growth conditions in $p$, we require $H$ to be continuous in $x$ in Section 2, and of Tonelli type in Section 3.

The main object of our investigation is the stochastic Hamilton-Jacobi equation

$$
H(x, D v(x, \omega), \omega)=c \quad \text { in } \mathbb{R}^{N}
$$

corresponding to the critical value $c$ of $H$. The latter is characterized by the property that Eq. (0.1) has admissible subsolutions, but $H=a$ does not at any subcritical level, i.e. whenever $a<c$. By admissible subsolution we mean a random Lipschitz function that has stationary increments, sublinear growth at infinity, and the property of being

\footnotetext{
* Corresponding author.

E-mail addresses: davini@mat.uniroma1.it (A. Davini), siconolf@mat.uniroma1.it (A. Siconolfi).
} 
an almost everywhere subsolution in $\mathbb{R}^{N}$, almost surely with respect to $\omega$. By convexity of the Hamiltonian, this last condition is equivalent to the notion of viscosity subsolution.

It is well known that the critical value is the unique level for which the corresponding Hamilton-Jacobi equation may have viscosity solutions, also termed correctors for the role they play in associated homogenization problems. The issue of finding characterizing conditions for the existence of correctors has been addressed in $[10,11]$ by means of a stochastic version of weak KAM Theory and of an adaptation of the metric techniques developed for deterministic Hamilton-Jacobi equations. The cornerstone of this approach is a random notion of Aubry set $\mathcal{A}(\omega)$, defined following the basic idea that the real invariant objects to look at in the analysis of stationary ergodic equations are not any more single points of the state space but instead random closed stationary sets. The set $\mathcal{A}(\omega)$ has been characterized in the aforementioned references as the minimal closed stationary random set for which there exists an admissible subsolution strict in its complement, at least in a weak sense, see Section 2.1 for precise definitions and results.

The present paper fits into the same line of research. It specifically aims at generalizing the previous characterization in two directions. On one side we would like to get admissible critical subsolutions, strict outside the random Aubry set in a stronger more classical sense; namely, we look for critical admissible subsolutions $v$ satisfying

$$
H(x, D v(x, \omega), \omega) \leq c-\delta \text { for a.e. } x \in U,
$$

for every open set $U$ compactly contained in $\mathbb{R}^{N} \backslash \mathcal{A}(\omega)$ and for every $\omega$ in a set of probability 1 , with $\delta>0$ depending on $U$ and $\omega$. On the other side, assuming the Hamiltonian of Tonelli type, we would like to show that such random functions can be taken almost surely of class $C^{1,1}$ in the whole $\mathbb{R}^{N}$. Both these goals are achieved by making use of the properties of the associated positive and negative Lax-Oleinik semigroups, see Section 1.4 for the precise definitions. This is new in this context and shows the effectiveness of these tools in the stationary ergodic setting, opening the door to a fruitful use of it for the study of other topics in the field, as well.

The sought generalizations can be also regarded as a step forward to deduce comparison principles for the critical equation. In the periodic case, for instance, similar results permit to show that the Aubry set is a uniqueness set for the critical equation, meaning that two admissible solutions agreeing on it are in fact the same. Here the problem gets more involved and remains open since it is not still clear how to exploit the weak form of compactness encoded in the stationary ergodic model.

The connection between regularity of subsolutions and the property of being strict in some distinguished regions can be understood if we think of finding regular subsolutions of the Hamilton-Jacobi equation at a supercritical level. In this case, the problem becomes relatively easy and does not require any special theory to be developed since, roughly speaking, there is some space to make a direct regularization starting from any globally strict subsolution, for example through mollification, without violating the subsolution property. When instead no strict subsolutions are available to start with, the problem becomes difficult and requires a deeper understanding of the setup.

To give account of the main results on the subject in the deterministic case, we recall that an initial crucial step has been to realize that the obstruction in getting strict subsolution for critical equations is not spread out indistinctly on the whole ambient space, but is concentrated around a specific set named after Aubry. Using this information, the existence of $C^{1}$ critical subsolutions has been first proved in [20] for Tonelli Hamiltonians and subsequently in [21] for Hamiltonians Lipschitz-continuous in the state variable through a technique combining partitions of unity and coverings, which non-surprisingly requires quite laborious estimates in proximity of the Aubry set.

Next, a relevant progress has been made in [3] where $C^{1,1}$ subsolutions, which is the optimal attainable regularity, have been found, at least when the Hamiltonian is Tonelli and the ambient space compact, through a simpler and more powerful procedure based on a double alternate application of positive and negative Lax-Oleinik semigroup. Within this approach, the difficulty of dealing with the Aubry set is bypassed thanks to the fact that the action of such semigroups do not affect critical subsolutions on the Aubry set. A nontrivial extension of this result to noncompact setting has been more recently provided in [19] by means of countable many alternative applications of Lax-Oleinik semigroups.

To further illustrate our results avoiding technical complications, we assume in the remainder of this introduction the critical value to be 0 , which is not restrictive up to adding a constant to the Hamiltonian. Our main achievements are the following: first, we provide a construction of the random Aubry set that simplifies the one given in [10] and that allows us to get rid of a restrictive condition therein assumed (see (A) in Section 2.1), still keeping the crucial property of existence of an admissible critical subsolution, weakly strict outside it. The crucial improvement with 
respect to the analysis performed in [10] is that the main tools used here are Lax-Oleinik semigroups instead of the critical semidistance.

Secondly, as already pointed out, we in addition establish the existence of admissible critical subsolutions that are strict, in the usual and stronger sense (0.2), outside the random Aubry set. More precisely, we show that any given weakly strict critical subsolution can be approximated, uniformly in $\mathbb{R}^{N}$, by a critical subsolution that is strict outside the random Aubry set, see Theorem 2.16. The key point here is the discovery of the fact that, when acting on a weakly strict subsolution, the negative Lax-Oleinik semigroup produces a 1-parameter family of admissible critical subsolutions that is strictly increasing outside the random Aubry set, see Proposition 2.19. In the end, the sought strict subsolution is defined, as usual in the topic, through infinite convex combination of the critical subsolutions obtained by applying the negative Lax-Oleinik semigroup to the initial weakly strict one at suitably small times, see Theorem 2.16. Via a standard argument, it is then easy to prove that strict critical subsolutions are dense, with respect to a suitable metric, among all critical admissible ones.

When the Hamiltonian is additionally assumed of Tonelli type, such strict critical subsolutions can be taken of class $C^{1,1}$ in $\mathbb{R}^{N}$. This is obtained by first deducing a further invariance of Lax-Oleinik semigroups with respect to strict critical subsolutions, and then by applying to the stationary ergodic environment the regularizing procedure due to Bernard, see [3]. The lack of compactness of the ground space does not affect the method since, under our assumptions, we have a global control on $\mathbb{R}^{N}$ of the semiconcavity or semiconvexity constants of the subsolutions generated in the construction, see Proposition 3.4.

The paper is divided in three sections. Section 1 has introductory character: we give notation, terminology and we recall some basic mathematical facts that will be used throughout the paper. Further we provide a brief presentation of the stationary ergodic setting and of the corresponding stochastic Hamilton-Jacobi equations, and present the salient properties of the positive and negative Lax-Oleinik semigroups in random environments. For the interpretation of the negative Lax-Oleinik semigroup stated in Proposition 1.13 we need a comparison principle for an associated timedependent Hamilton-Jacobi equation, which is proved in Appendix A. Section 2 is about continuous Hamiltonians. In the first subsection we give the definition of random Aubry set and we prove the existence of an admissible critical subsolution that is weakly strict outside it. In the second subsection we strengthen these results by showing existence and density of strict critical subsolutions. Section 3 is devoted to Hamiltonians of Tonelli type. In the first subsection we present some additional properties enjoyed by the Lax-Oleinik semigroups and by the random Aubry set in our setting, while in the second one we apply Bernard's method to the case at issue and we derive the announced results about regular strict subsolutions.

\section{Basic material}

\subsection{Notations and preliminaries}

We write below a list of symbols used throughout this paper.

$\begin{array}{ll}N & \text { an integer number } \\ B_{R}\left(x_{0}\right) & \text { the open ball in } \mathbb{R}^{N} \text { centered at } x_{0} \text { of radius } R \\ B_{R} & \text { the open ball in } \mathbb{R}^{N} \text { centered at } 0 \text { of radius } R \\ \langle\cdot, \cdot\rangle & \text { the scalar product in } \mathbb{R}^{N} \\ |\cdot| & \text { the Euclidean norm in } \mathbb{R}^{N} \\ \mathbb{R}_{+} & \text {the set of nonnegative real numbers } \\ \mathcal{B}\left(\mathbb{R}^{N}\right) & \text { the } \sigma \text {-algebra of Borel subsets of } \mathbb{R}^{N} \\ \chi_{E} & \text { the characteristic function of the set } E\end{array}$

Given a subset $U$ of $\mathbb{R}^{N}$, we denote by $\bar{U}$ its closure and by $\operatorname{int}(U)$ its interior. We furthermore say that $U$ is compactly contained in a subset $V$ of $\mathbb{R}^{N}$ if $\bar{U}$ is compact and contained in $V$. If $E$ is a Lebesgue measurable subset of $\mathbb{R}^{N}$, we denote by $|E|$ its $N$-dimensional Lebesgue measure, and qualify $E$ as negligible whenever $|E|=$ 0 . We say that a property holds almost everywhere (a.e. for short) on $\mathbb{R}^{N}$ if it holds up to a negligible set. Given 
a function $u$ defined in $\mathbb{R}^{N}$, we will write $u \in C^{1,1}\left(B_{r}\left(x_{0}\right)\right)$ to mean that $u$ is of class $C^{1,1}$ in $B_{r}\left(x_{0}\right)$. We will denote by $\operatorname{Lip}\left(u ; B_{r}\left(x_{0}\right)\right)$ and $\operatorname{Lip}\left(D u ; B_{r}\left(x_{0}\right)\right)$ the Lipschitz constant of $u$ and $D u$ in $B_{r}\left(x_{0}\right)$, respectively.

With the term curve we will refer to an absolutely continuous function from some given interval $[a, b]$ to $\mathbb{R}^{N}$.

Given a continuous function $u$ on $\mathbb{R}^{N}$, we will call subtangent (respectively, supertangent) of $u$ at $x_{0}$ a function $\psi$ of class $C^{1}$ in a neighborhood $U$ of $x_{0}$ such that $u-\psi$ has a local minimum (resp., maximum) at $x_{0}$. The (possibly empty) set made up by the differentials of supertangents (resp. subtangents) at $x_{0}$ is called superdifferential (resp. subdifferential) and denoted by $D^{+} u\left(x_{0}\right)$ (resp. $D^{-} u\left(x_{0}\right)$ ). We recall that if both super and subdifferential are nonempty then $u$ is differentiable at $x_{0}$.

Given a locally Lipschitz function $u$ in an open subset $U$ of $\mathbb{R}^{N}$, we will denote by $\partial^{*} u\left(x_{0}\right)$ the set of reachable gradients of $u$ at $x_{0}$, that is the set

$$
\partial^{*} u\left(x_{0}\right)=\left\{\lim _{n} D u\left(x_{n}\right): u \text { is differentiable at } x_{n}, x_{n} \rightarrow x_{0}\right\},
$$

while Clarke's generalized gradient $\partial^{c} u\left(x_{0}\right)$ is the closed convex hull of $\partial^{*} u\left(x_{0}\right)$. The set $\partial^{c} u\left(x_{0}\right)$ contains both $D^{+} u\left(x_{0}\right)$ and $D^{-} u\left(x_{0}\right)$, in particular $D u\left(x_{0}\right) \in \partial^{c} u\left(x_{0}\right)$ at any differentiability point $x_{0}$ of $u$. We recall that the setvalued map $x \mapsto \partial^{c} u(x)$ is upper semicontinuous with respect to set inclusion. When $\partial^{c} u\left(x_{0}\right)$ reduces to a singleton, the function $u$ is said to be strictly differentiable at that point. In this instance, $u$ is differentiable at $x_{0}$ and its gradient is continuous at $x_{0}$. When $u$ depends on a time and space variable, indicated by $t$ and $x$, respectively, we will denote by $\partial_{t}^{c} u(x, t)$ Clarke's generalized gradient of the function $u(\cdot, x)$ at $t$, and by $\partial_{x}^{c} u(x, t)$ Clarke's generalized gradient of $u(t, \cdot)$ at $x$. We refer the reader to [6] for a detailed treatment of the subject.

A function $u$ will be said to be semiconcave on an open subset $U$ of $\mathbb{R}^{N}$ if for every $x \in U$ there exists a vector $p_{x} \in \mathbb{R}^{N}$ such that

$$
u(y)-u(x) \leqslant\left\langle p_{x}, y-x\right\rangle+|y-x| \Theta(|y-x|) \text { for every } y \in U,
$$

where $\Theta$ is a modulus. The vectors $p_{x}$ satisfying such inequality are precisely the elements of $D^{+} u(x)$, which is thus always nonempty in $U$. Moreover, $\partial^{c} u(x)=D^{+} u(x)$ for every $x \in U$, yielding in particular that $D u$ is continuous on its domain of definition in $U$, see [5]. Finally, we say that a function $u$ is semiconvex if $-u$ is semiconcave.

Throughout the paper, $(\Omega, \mathcal{F}, \mathbb{P})$ will denote a separable probability space, where $\mathbb{P}$ is the probability measure and $\mathcal{F}$ the $\sigma$-algebra of $\mathbb{P}$-measurable sets. Here separable is understood in the measure theoretic sense, meaning that the Hilbert space $L^{2}(\Omega)$ is separable, cf. [27] also for other equivalent definitions. A property will be said to hold almost surely (a.s. for short) on $\Omega$ if it holds up to a subset of probability 0 . We will indicate by $L^{p}(\Omega), p \geq 1$, the usual Lebesgue space on $\Omega$ with respect to $\mathbb{P}$. If $f \in L^{1}(\Omega)$, we write $\mathbb{E}(f)$ for the mean of $f$ on $\Omega$, i.e. the quantity $\int_{\Omega} f(\omega) \mathrm{d} \mathbb{P}(\omega)$.

We qualify as measurable a map from $\Omega$ to itself, or to a topological space $\mathcal{M}$ with Borel $\sigma$-algebra $\mathcal{B}(\mathcal{M})$, if the inverse image of any set in $\mathcal{F}$ or in $\mathcal{B}(\mathcal{M})$ belongs to $\mathcal{F}$. The latter will be also called random variable with values in $\mathcal{M}$.

We will be interested in the case when the range of a random variable is the Polish space (i.e. a complete and separable metric space) $\mathrm{C}\left(\mathbb{R}^{N}\right)$ of continuous real functions on $\mathbb{R}^{N}$, endowed with a metric $d$ inducing the topology of uniform convergence on compact subsets of $\mathbb{R}^{N}$. It can be for instance defined by

$$
d(f, g):=\sum_{n=1}^{\infty} \frac{1}{2^{n}} \frac{\|f-g\|_{L^{\infty}\left(B_{n}\right)}}{\|f-g\|_{L^{\infty}\left(B_{n}\right)}+1} \quad f, g \in \mathrm{C}\left(\mathbb{R}^{k}\right) .
$$

We will furthermore denote by $\operatorname{Lip}_{\vartheta}\left(\mathbb{R}^{n}\right)$ the subspace of $\mathrm{C}\left(\mathbb{R}^{N}\right)$ made up of Lipschitz-continuous real functions with Lipschitz constant less than or equal to $\vartheta>0$. We will briefly say Lipschitz random function to mean a $\vartheta$-Lipschitz random function for some $\vartheta>0$.

Let $\left(f_{n}\right)_{n}$ be a sequence of random variables taking values in $\mathrm{C}\left(\mathbb{R}^{N}\right)$. We will say that $f_{n}$ converge to $f$ in probability if, for every $\varepsilon>0$,

$$
\mathbb{P}\left(\left\{\omega \in \Omega: d\left(f_{n}(\omega), f(\omega)\right)>\varepsilon\right\}\right) \rightarrow 0 \quad \text { as } n \rightarrow+\infty .
$$

The limit $f$ is still a random variable. Since $\mathrm{C}\left(\mathbb{R}^{N}\right)$ is a separable metric space, almost sure convergence, i.e. $d\left(f_{n}(\omega), f(\omega)\right) \rightarrow 0$ a.s. in $\omega$, implies convergence in probability, while the converse is not true in general. However, the following characterization holds: 
Theorem 1.1. Let $f_{n}$, $f$ be random variables with values in $\mathrm{C}\left(\mathbb{R}^{N}\right)$. Then $f_{n} \rightarrow f$ in probability if and only if every subsequence $\left(f_{n_{k}}\right)_{k}$ has a subsequence converging to $f$ a.s.

We denote by $L^{0}\left(\Omega ; \mathrm{C}\left(\mathbb{R}^{N}\right)\right)$ the space made up by the equivalence classes of random variables with values in $\mathrm{C}\left(\mathbb{R}^{N}\right)$ for the relation of almost sure equality. For every $f, g \in L^{0}\left(\Omega ; \mathrm{C}\left(\mathbb{R}^{N}\right)\right)$, we set

$$
\mu(f, g):=\inf \{\varepsilon \geq 0: \mathbb{P}(\{\omega \in \Omega: d(f(\omega), g(\omega))>\varepsilon\}) \leq \varepsilon\} .
$$

Theorem 1.2. $\mu$ is a metric, named after Ky Fan, which metrizes convergence in probability, i.e. $\mu\left(f_{n}, f\right) \rightarrow 0$ if and only if $f_{n} \rightarrow f$ in probability, and turns $L^{0}\left(\Omega ; \mathrm{C}\left(\mathbb{R}^{N}\right)\right)$ into a Polish space.

\subsection{Stationary ergodic setting}

An $N$-dimensional dynamical system $\left(\tau_{x}\right)_{x \in \mathbb{R}^{N}}$ is defined as a family of mappings $\tau_{x}: \Omega \rightarrow \Omega$ which satisfy the following properties:

(1) the group property: $\tau_{0}=i d, \tau_{x+y}=\tau_{x} \circ \tau_{y}$;

(2) the mappings $\tau_{x}: \Omega \rightarrow \Omega$ are measurable and measure preserving, i.e. $\mathbb{P}\left(\tau_{x} E\right)=\mathbb{P}(E)$ for every $E \in \mathcal{F}$;

(3) the map $(x, \omega) \mapsto \tau_{x} \omega$ from $\mathbb{R}^{N} \times \Omega$ to $\Omega$ is jointly measurable, i.e. measurable with respect to the product $\sigma$-algebra $\mathcal{B}\left(\mathbb{R}^{N}\right) \otimes \mathcal{F}$.

We will moreover assume that $\left(\tau_{x}\right)_{x \in \mathbb{R}^{N}}$ is ergodic, i.e. that one of the following equivalent conditions hold:

(i) every measurable function $f$ defined on $\Omega$ such that, for every $x \in \mathbb{R}^{N}, f\left(\tau_{x} \omega\right)=f(\omega)$ a.s. in $\Omega$, is almost surely constant;

(ii) every set $A \in \mathcal{F}$ such that $\mathbb{P}\left(\tau_{x} A \Delta A\right)=0$ for every $x \in \mathbb{R}^{N}$ has probability either 0 or 1 , where $\Delta$ stands for the symmetric difference.

Given a random variable $f: \Omega \rightarrow \mathbb{R}$, for any fixed $\omega \in \Omega$ the function $x \mapsto f\left(\tau_{x} \omega\right)$ is said to be a realization of $f$. The following properties follow from Fubini's Theorem, see [22]: if $f \in L^{p}(\Omega)$, then $\mathbb{P}$-almost all its realizations belong to $L_{l o c}^{p}\left(\mathbb{R}^{N}\right)$; if $f_{n} \rightarrow f$ in $L^{p}(\Omega)$, then $\mathbb{P}$-almost all realizations of $f_{n}$ converge to the corresponding realization of $f$ in $L_{l o c}^{p}\left(\mathbb{R}^{N}\right)$. The Lebesgue spaces on $\mathbb{R}^{N}$ are understood with respect to the Lebesgue measure.

A jointly measurable function $v$ defined in $\mathbb{R}^{N} \times \Omega$ is said stationary if, for every $z \in \mathbb{R}^{N}$, there exists a set $\Omega_{z}$ with probability 1 such that for every $\omega \in \Omega_{z}$

$$
v(\cdot+z, \omega)=v\left(\cdot, \tau_{z} \omega\right) \quad \text { on } \mathbb{R}^{N}
$$

With the term (graph-measurable) random set we indicate a set-valued function $X: \Omega \rightarrow \mathcal{B}\left(\mathbb{R}^{N}\right)$ with

$$
\Gamma(X):=\left\{(x, \omega) \in \mathbb{R}^{N} \times \Omega: x \in X(\omega)\right\}
$$

jointly measurable in $\mathbb{R}^{N} \times \Omega$. A random set $X$ will be qualified as stationary if for every for every $z \in \mathbb{R}^{N}$, there exists a set $\Omega_{z}$ of probability 1 such that

$$
X\left(\tau_{z} \omega\right)=X(\omega)-z \quad \text { for every } \omega \in \Omega_{z} .
$$

We use a stronger notion of measurability, which is usually named in the literature after Effros, to define a closed random set, say $X(\omega)$. Namely we require $X(\omega)$ to be a closed subset of $\mathbb{R}^{N}$ for any $\omega$ and

$$
\{\omega: X(\omega) \cap K \neq \varnothing\} \in \mathcal{F}
$$

with $K$ varying among the compact (equivalently, open) subsets of $\mathbb{R}^{N}$. If $X(\omega)$ is measurable in this sense then it is also graph-measurable, see [24] for more details.

A closed random set $X$ is called stationary if it, in addition, satisfies (1.2). Note that in this event the set $\{\omega$ : $X(\omega) \neq \varnothing\}$, which is measurable by the Effros measurability of $X$, is invariant with respect to the group of translations $\left(\tau_{x}\right)_{x \in \mathbb{R}^{N}}$ by stationarity, so it has probability either 0 or 1 by the ergodicity assumption. 
The following holds, see [12]:

Proposition 1.3. Let $f$ be a continuous random function and $C$ a closed subset of $\mathbb{R}$. Then

$$
X(\omega):=\{x: f(x, \omega) \in C\}
$$

is a closed random set in $\mathbb{R}^{N}$. If in addition $f$ is stationary, then $X$ is stationary.

Definition 1.4. A random Lipschitz function $v$ is said to have stationary increments if, for every $z \in \mathbb{R}^{N}$, there exists a set $\Omega_{z}$ of probability 1 such that

$$
v(x+z, \omega)-v(y+z, \omega)=v\left(x, \tau_{z} \omega\right)-v\left(y, \tau_{z} \omega\right) \quad \text { for all } x, y \in \mathbb{R}^{N}
$$

for every $\omega \in \Omega_{z}$. This is equivalent to requiring that there exists a random variable $k(\omega)$, depending on $z$, for which

$$
v(\cdot+z, \omega)=v\left(\cdot, \tau_{z} \omega\right)+k(\omega) \quad \text { on } \mathbb{R}^{N} \text { for every } \omega \in \Omega_{z} .
$$

Let $v$ be a Lipschitz random function with stationary increments. For every fixed $x \in \mathbb{R}^{N}$, the random variable $D v(x, \cdot)$ is well defined on a set of probability 1, see [11] for the details. Accordingly, we can define the mean $\mathbb{E}(D v(x, \cdot))$, which is furthermore independent of $x$ by the stationary character of $D v$. We are interested in the case when this mean is zero.

Definition 1.5. A Lipschitz random function will be called admissible if it has stationary increments and gradient with mean 0 .

We state a characterizations of admissible random functions and a result that guarantees that stationary Lipschitz random functions are admissible, see [11] for the proofs.

Theorem 1.6. A Lipschitz random function $v$ with stationary increments has gradient with vanishing mean if and only if it is almost surely sublinear at infinity, namely

$$
\lim _{|x| \rightarrow+\infty} \frac{v(x, \omega)}{|x|}=0 \quad \text { a.s. in } \omega .
$$

Theorem 1.7. Any stationary Lipschitz random function $v$ is admissible.

\subsection{Stochastic Hamilton-Jacobi equations}

We consider a jointly measurable Hamiltonian

$$
H: \mathbb{R}^{N} \times \mathbb{R}^{N} \times \Omega \rightarrow \mathbb{R}
$$

satisfying the following conditions:

(H1) $H(\cdot, \cdot, \omega) \in \mathrm{C}\left(\mathbb{R}^{N} \times \mathbb{R}^{N}\right)$ for every $\omega$;

(H2) $H(x, \cdot, \omega)$ is convex on $\mathbb{R}^{N}$ for every $(x, \omega) \in \mathbb{R}^{N} \times \Omega$;

(H3) there exist two superlinear functions $\alpha_{H}, \beta_{H}: \mathbb{R}_{+} \rightarrow \mathbb{R}$ such that

$$
\alpha_{H}(|p|) \leq H(x, p, \omega) \leq \beta_{H}(|p|) \quad \text { for all }(x, p, \omega) \in \mathbb{R}^{N} \times \mathbb{R}^{N} \times \Omega ;
$$

$$
H(\cdot+z, \cdot, \omega)=H\left(\cdot, \cdot, \tau_{z} \omega\right) \text { for every }(z, \omega) \in \mathbb{R}^{N} \times \Omega \text {. }
$$

Remark 1.8. The functions $\alpha_{H}$ and $\beta_{H}$ appearing above can be taken convex and nondecreasing, without any loss of generality. This will be tacitly assumed in the sequel. Condition (H3) is equivalent to saying that $H$ is superlinear and locally bounded in $p$, uniformly with respect to $(x, \omega)$. We deduce from $(\mathrm{H} 2)$

$$
|H(x, p, \omega)-H(x, q, \omega)| \leq \tilde{M}_{R}|p-q| \text { for all } x, \omega \text {, and } p, q \text { in } B_{R},
$$

where $\widetilde{M}_{R}=\sup \left\{|H(x, p, \omega)|:(x, \omega) \in \mathbb{R}^{N} \times \Omega,|p| \leq R+2\right\}$, which is finite thanks to (H3). 
For every $a \in \mathbb{R}$, we are interested in the stochastic Hamilton-Jacobi equation

$$
H(x, D v(x, \omega), \omega)=a \quad \text { in } \mathbb{R}^{N} .
$$

The material we are about to expose has been already presented in [10-12], to which we refer for the details. Here we just recall the main items.

We say that a Lipschitz random function $v$ is a solution (resp. subsolution) of $(1.7)$ if $v(\cdot, \omega)$ is a viscosity solution (resp. subsolution) of (1.7) for every $\omega$ in a set of probability 1 . We recall that, due to the convexity assumption (H2), the notion of almost everywhere subsolution is equivalent to the one in the viscosity sense. We refer the reader to [1,2] for more details on the theory of viscosity solutions in the deterministic case. In particular, any such subsolution is almost surely in $\operatorname{Lip}_{\kappa_{a}}\left(\mathbb{R}^{n}\right)$, where

$$
\kappa_{a}:=\sup \left\{|p|: H(x, p, \omega) \leq a \text { for some }(x, \omega) \in \mathbb{R}^{N} \times \Omega\right\},
$$

which is finite thanks to (H3). We are interested in the class of admissible subsolutions, hereafter denoted by $\mathcal{S}_{a}$, i.e. random functions taking values in $\operatorname{Lip}_{\kappa_{a}}(\mathbb{R})$ with stationary increments and zero mean gradient that are subsolutions of (1.7). An admissible solution will be also named exact corrector, remembering its role in homogenization.

We proceed by defining the free and the stationary random critical value, denoted by $c_{f}(\omega)$ and $c$ respectively, as follows:

$$
\begin{aligned}
c_{f}(\omega) & =\inf \left\{a \in \mathbb{R}:(1.7) \text { has a subsolution } v \in \operatorname{Lip}\left(\mathbb{R}^{N}\right)\right\}, \\
c & =\inf \left\{a \in \mathbb{R}: \mathcal{S}_{a} \neq \emptyset\right\} .
\end{aligned}
$$

We emphasize that in definition (1.9) we are considering deterministic a.e. subsolutions $v$ of Eq. (1.7), where $\omega$ is treated as a fixed parameter. Furthermore, we note that $c_{f}\left(\tau_{z} \omega\right)=c_{f}(\omega)$ for every $(z, \omega) \in \mathbb{R}^{N} \times \Omega$, so that, by ergodicity, the random variable $c_{f}(\omega)$ is almost surely equal to a constant, still denoted by $c_{f}$. Hereafter we will write $\Omega_{f}$ for the set of probability 1 where $c_{f}(\omega)$ equals $c_{f}$.

It is apparent by the definitions that $c \geq c_{f}$. The relation of these two values with the effective Hamiltonian obtained via the homogenization $[25,26]$ is discussed in $[23,11]$.

In the sequel, we will focus our attention on the critical equation

$$
H(x, D v(x, \omega), \omega)=c \quad \text { in } \mathbb{R}^{N} .
$$

The following result holds, see $[23,11]$ :

Theorem 1.9. There exist admissible critical subsolutions, i.e. $\mathcal{S}_{c} \neq \emptyset$.

Moreover, the critical equation is the only equation of the kind (1.7) for which exact correctors may exist, see [12].

We recall the main items of the so called metric method for Hamilton-Jacobi equations, as adapted in [11] to the case at issue. In next formulae we assume that $a \geq c_{f}$ and $\omega \in \Omega_{f}$. Let

$$
Z_{a}(x, \omega):=\{p: H(x, p, \omega) \leq a\},
$$

be the $a$-sublevels of the Hamiltonian and

$$
\sigma_{a}(x, q, \omega):=\sup \left\{\langle q, p\rangle: p \in Z_{a}(x, \omega)\right\}
$$

the related support functions. It comes from (1.6) (cf. Lemma 4.6 in [12]) that, given $b>a$, we can find $\rho=$ $\rho(b, a)>0$ with

$$
Z_{a}(x, \omega)+B_{\rho} \subseteq Z_{b}(x, \omega) \text { for every }(x, \omega) \in \mathbb{R}^{N} \times \Omega_{f} .
$$

It is straightforward to check that $\sigma_{a}$ is convex in $q$, upper semicontinuous in $x$ and, in addition, continuous whenever $Z_{a}(x, \omega)$ has nonempty interior or reduces to a point. We extend the definition of $\sigma_{a}$ to $\mathbb{R}^{N} \times \mathbb{R}^{N} \times \Omega$ by setting $\sigma_{a}(\cdot, \cdot, \omega) \equiv 0$ for every $\omega \in \Omega \backslash \Omega_{f}$. With this choice, the function $\sigma_{a}$ is jointly measurable in $\mathbb{R}^{N} \times \mathbb{R}^{N} \times \Omega$ and enjoys the stationarity property

$$
\sigma_{a}(\cdot+z, \cdot, \omega)=\sigma_{a}\left(\cdot, \cdot, \tau_{z} \omega\right) \quad \text { for every } z \in \mathbb{R}^{N} \text { and } \omega \in \Omega .
$$


We define the semidistance $S_{a}$ as

$$
S_{a}(x, y, \omega)=\inf \left\{\int_{0}^{1} \sigma_{a}(\gamma(s), \dot{\gamma}(s), \omega) \mathrm{d} s: \gamma \in \operatorname{Lip}_{x, y}\left([0,1] ; \mathbb{R}^{N}\right)\right\} .
$$

The following holds, cf. [21]:

Proposition 1.10. Let $a \geq c_{f}$ and $\omega \in \Omega_{f}$. We have:

(i) For any $y \in \mathbb{R}^{N}$, the function $S_{a}(y, \cdot, \omega)$ is a subsolution of $(1.7)$ in $\mathbb{R}^{N}$, and also a solution in $\mathbb{R}^{N} \backslash\{y\}$.

(ii) A continuous function $\phi$ is a subsolution of (1.7) if and only if

$$
\phi(x)-\phi(y) \leq S_{a}(y, x, \omega) \text { for all } x, y \in \mathbb{R}^{N} .
$$

\subsection{Positive and negative Lax-Oleinik semigroups}

The Lagrangian associated with $H$ by duality is the function defined as

$$
L(x, q, \omega):=\max _{p \in \mathbb{R}^{N}}\{\langle p, q\rangle-H(x, p, \omega)\} \quad \text { for all }(x, q, \omega) \in \mathbb{R}^{N} \times \mathbb{R}^{N} \times \Omega .
$$

As well known, the Lagrangian satisfies properties analogous to (H1)-(H4).

For every $t>0$ and $\omega \in \Omega$, we define a function $h_{t}$ on $\mathbb{R}^{N} \times \mathbb{R}^{N}$ as

$$
h_{t}(x, y, \omega):=\inf \left\{\int_{0}^{t} L(\gamma, \dot{\gamma}, \omega) \mathrm{d} s: \gamma(0)=x, \gamma(t)=y\right\} .
$$

Curves that realize the above infimum are called Lagrangian minimizers. It is well known that such minimizers always exist and they are Lipschitz continuous, see [4].

It is known, see for instance [16], that

$$
S_{a}(y, x, \omega)=\inf _{t>0}\left(h_{t}(y, x, \omega)+a t\right)
$$

for every $a \in \mathbb{R}$ and $\omega \in \Omega_{f}$. In particular, when $\omega \in \Omega_{f}$ and $a \geq c_{f}$, we derive, in view of Proposition 1.10, that a function $v$ is a subsolution of (1.7) if and only if

$$
v(x)-v(y) \leq h_{t}(x, y)+a t \quad \text { for every } x, y \in \mathbb{R}^{N} \text { and } t>0 .
$$

For every $t>0$ and every $\kappa$-Lipschitz random function $u$, we next define the negative and positive Lax-Oleinik semigroups $T_{t}^{-} u, T_{t}^{+} u$ as follows:

$$
\begin{aligned}
& \left(T_{t}^{-} u\right)(x, \omega):=\inf \left\{u(y, \omega)+h_{t}(y, x, \omega): y \in \mathbb{R}^{N}\right\} \\
& \left(T_{t}^{+} u\right)(x, \omega):=\sup \left\{u(y, \omega)-h_{t}(x, y, \omega): y \in \mathbb{R}^{N}\right\}
\end{aligned}
$$

for every $(x, \omega) \in \mathbb{R}^{N} \times \Omega$.

Remark 1.11. It is worth noticing that $T_{t}^{+} u=-\check{T}_{t}^{-}(-u)$, where we have denoted by $\check{T}_{t}^{-}$the negative Lax-Oleinik semigroup associated with the Hamiltonian $\check{H}$ defined by

$$
\check{H}(x, p, \omega):=H(x,-p, \omega) \text { for every }(x, p, \omega) \in \mathbb{R}^{N} \times \mathbb{R}^{N} \times \Omega .
$$

We list a series of properties enjoyed by such semigroups.

Proposition 1.12. Let $\vartheta>0$. Then there exists $R(\vartheta)>0$ such that for every $\vartheta$-Lipschitz random function $u$ and every $\omega \in \Omega$ the following properties hold: 
(i)

$$
\begin{aligned}
& \left(T_{t}^{-} u\right)(x, \omega)<\inf \left\{u(y, \omega)+h_{t}(y, x, \omega):|x-y| \geq t R(\vartheta)\right\}, \\
& \left(T_{t}^{+} u\right)(x, \omega)>\sup \left\{u(y, \omega)-h_{t}(y, x, \omega):|x-y| \geq t R(\vartheta)\right\} ;
\end{aligned}
$$

(ii) the maps $(t, x) \mapsto T_{t}^{ \pm} u(x, \omega)$ are $R(\vartheta)$-Lipschitz continuous on $[0,+\infty) \times \mathbb{R}^{N}$. In particular

$$
\left\|T_{t}^{ \pm} u(\cdot, \omega)-u(\cdot, \omega)\right\|_{\infty} \leq t R(\vartheta) \quad \text { for every } t>0
$$

(iii) if $u$ has stationary increments (resp. is stationary), then $T_{t}^{ \pm} u$ have stationary increments (resp. are stationary) for any $t>0$.

Items (i), (ii) are the ergodic stationary version of well known estimates holding in deterministic case. Bounding function $R(\cdot)$ solely depends on the $\alpha_{H}(\cdot), \beta_{H}(\cdot)$ appearing in (H3), which are invariant with respect to $\omega$. Item (iii) is straightforward.

The next two results are purely deterministic, with $\omega$ thought as a fixed parameter. The first one is classical, see for instance $[17,15]$ for the proof.

Proposition 1.13. Let $u_{0}$ be a Lipschitz, random function. Then, for every fixed $\omega \in \Omega$, the function $u(t, x):=$ $\left(T_{t}^{-} u_{0}\right)(x, \omega)$ is the unique Lipschitz continuous viscosity solution of the time-dependent equation

$$
\partial_{t} u+H\left(x, D_{x} u, \omega\right)=0 \quad \text { in }(0,+\infty) \times \mathbb{R}^{N}
$$

satisfying $u(0, x)=u_{0}(x, \omega)$ on $\mathbb{R}^{N}$.

The fact that $u(t, x):=\left(T_{t}^{-} u_{0}\right)(x, \omega)$ is a Lipschitz solution of $(1.16)$ assuming the initial datum $u_{0}(\cdot, \omega)$ is well known, see for instance $[1,8,9]$. The fact that it is unique is usually obtained from a comparison principle between uniformly continuous sub and supersolutions of (1.16), which is proved under the additional assumption that $H(\cdot, \cdot, \omega)$ is uniformly continuous in domains of the form $\mathbb{R}^{N} \times B_{R}$, for every $R>0$, see for instance [15]. Since we do not want to strengthen our hypotheses on $H$, we show in Appendix A that such a comparison principle keeps holding in our setting if we restrict to Lipschitz sub and supersolutions, see Theorem A.1.

Sub and supersolutions of the stationary equation (1.7) can be characterized through the action of the negative Lax-Oleinik semigroup as follows:

Proposition 1.14. Let $v$ be a Lipschitz continuous function on $\mathbb{R}^{N}$ and let $\omega$ be fixed. The following facts hold:

(i) $v(\cdot, \omega)$ is a subsolution of (1.7) if and only if $t \mapsto T_{t}^{-} v(\cdot, \omega)+$ at is non-decreasing;

(ii) $v(\cdot, \omega)$ is a solution of (1.7) if and only if $v \equiv T_{t}^{-} v(\cdot, \omega)+$ at for every $t>0$.

Proof. Let us prove (i). If $v(\cdot, \omega)$ is a subsolution of (1.7), then the function $(t, x) \mapsto v(x, \omega)-a t$ is a subsolution of (1.16). From Proposition 1.13 and Theorem A.1 we infer that $v(\cdot, \omega)-a t \leq T_{t}^{-} v(\cdot, \omega)$ for every $t>0$ and the assertion follows in view of the monotone character of the semigroup $T_{t}^{-}$. Conversely, if $t \mapsto T_{t}^{-} v(\cdot, \omega)+a t$ is non-decreasing, we get in particular that $v(\cdot, \omega)$ enjoys (1.15), i.e. it is a subsolution of (1.7). Assertion (ii) readily follows from Proposition 1.13 by noticing that $v$ is a solution of (1.7) if and only if $v(x)-a t$ is a solution of (1.16) with initial datum $u_{0}:=v$.

As a consequence of the previous results, we deduce the following fact:

Proposition 1.15. Let $u \in \mathcal{S}_{a}$. Then both $T_{t}^{+} u$ and $T_{t}^{-} u$ belong to $\mathcal{S}_{a}$, for every $t>0$.

\section{Continuous Hamiltonians}

In this section we assume $H$ to satisfy assumptions (H1)-(H4). In the first subsection we give the definition of random Aubry set and we prove the existence of a critical admissible subsolution that is weakly strict outside it, in 
a sense that will be clarified below, see Definition 2.3. This can be regarded as a completion of [10], where analogous results were obtained under an additional assumption, see (A) below. In the second subsection we show how a strict admissible critical subsolution can be produced starting from a weakly strict one. For the outcomes of both subsections, we essentially use the (negative) Lax-Oleinik semigroup and its properties.

From now on we will assume the stationary critical value $c=0$, which is not restrictive up to replacing $H$ with $H-c$, and focus our analysis on such critical level. To ease notation, we will omit the subscript $c$ when no ambiguity is possible. In particular, we will simply write $\mathcal{S}, S, \kappa$ and $\sigma$ in place of $\mathcal{S}_{c}, S_{c}, \kappa_{c}$ and $\sigma_{c}$.

\subsection{Aubry set and weakly strict subsolutions}

We start by recalling the definition of the classical Aubry set.

Definition 2.1. The classical Aubry set $\mathcal{A}_{f}(\omega)$ is the closed stationary random set defined as follows:

$$
\mathcal{A}_{f}(\omega)=\left\{y \in \mathbb{R}^{N}: \liminf _{t \rightarrow+\infty} h_{t}(y, y, \omega)=0\right\} \quad \text { for any } \omega \in \Omega .
$$

The random set $\mathcal{A}_{f}(\omega)$ is almost surely empty whenever the random critical value is strictly greater than $c_{f}$, i.e. when $0>c_{f}$. When $c_{f}=0, \mathcal{A}_{f}(\omega)$ may be either almost surely nonempty or almost surely empty. This latter instance is not specific of the stationary ergodic setting: it can occur even in the periodic case.

We proceed by presenting the notion of random Aubry set as introduced in [10]. For this, we need to recall some facts about Lax formulae in the stationary ergodic setting, see [11,12] for more details. For any given almost surely nonempty stationary closed random set $C(\omega)$ in $\mathbb{R}^{N}$ and any critical subsolution $g \in \mathcal{S}$, the Lax formula is given by

$$
u(x, \omega):=\inf \{g(y, \omega)+S(y, x, \omega): y \in C(\omega)\} \quad x \in \mathbb{R}^{N},
$$

and provides another admissible critical subsolution enjoying some additional properties, see Proposition 4.1 in [10]. More precisely, $u(\cdot, \omega)$ is the maximal critical subsolution agreeing with $g(\cdot, \omega)$ on $C(\omega)$ a.s. in $\omega$, and, as a consequence

$$
u(\cdot, \omega) \text { is a critical solution in } \mathbb{R}^{N} \backslash C(\omega) \text { a.s. in } \omega .
$$

In the above formula we agree that $u(\cdot, \omega) \equiv 0$ when either $C(\omega)=\emptyset$ or the infimum is equal to $-\infty$.

Inspired by the periodic model case, we define the stationary random Aubry set as follows:

Definition 2.2. A stationary and almost surely nonempty closed random set $C(\omega)$ will be called random Aubry set and will be denoted by $\mathcal{A}(\omega)$ if

(i) the extension of any admissible critical subsolution from $C(\omega)$ via the Lax formula (2.1) yields an admissible critical solution;

(ii) any stationary and almost surely nonempty closed random set that satisfies the previous property is almost surely contained in $C(\omega)$.

If there are no stationary and almost surely nonempty closed random sets satisfying (i), we agree that the Aubry set is almost surely empty.

We now proceed to show that the above definition is well posed. For this, we will take on the burden of checking that, if there is some nonempty stationary closed random set satisfying item $(i)$ above, then there is also a maximal one. This task is by no means trivial, the main difficulty being the following: the random sets needed for the construction cannot be defined by treating $\omega$ as a fixed parameter because this procedure would lead to objects that do not satisfy the proper measurability hypotheses.

We also underline that the case of an almost surely empty random Aubry set (i.e. the case of nonexistence of nonempty stationary closed random sets satisfying item (i) above) can actually occur, see for instance Example 4.10 in [11]. 
The fact that Definition 2.2 is well-posed has been already shown in [10] under the following additional assumption:

$$
\text { either } c_{f}<0 \text { or } c_{f}=0 \text { and } \mathcal{A}_{f}(\omega)=\emptyset \quad \text { a.s. in } \omega \text {. }
$$

As already announced, the novelty here is that we get rid of (A) by using the negative Lax-Oleinik semigroup.

In order to state the result we aim at, we need the following

Definition 2.3. Let $C(\omega)$ be a stationary closed random set. A critical subsolution $v \in \mathcal{S}$ is said to be weakly strict in $\mathbb{R}^{N} \backslash C(\omega)$ if the following property holds a.s. in $\omega$ :

$$
v(x, \omega)-v(y, \omega)<S(y, x, \omega) \text { for every } x, y \in \mathbb{R}^{N} \backslash C(\omega) \text { with } x \neq y .
$$

Remark 2.4. If $v \in \mathcal{S}$ is a weakly strict critical subsolution in $\mathbb{R}^{N} \backslash C(\omega)$, the inequality (2.3) keeps holding even if one (but only one) between the points $x, y$ belongs to $C(\omega)$. This can be deduced from the fact that $S(\cdot, \cdot, \omega)$ is a geodesic-type semidistance. Namely, assume for definiteness that $x \notin C(\omega)$ and choose $r>0$ such that the closed ball $\overline{B_{r}}(x)$ is disjoint from $C(\omega)$ and does not contain $y$. By definition of the critical semidistance, it is easily seen that

$$
S(y, x, \omega)=\min _{z \in \partial B_{r}(x)} S(y, z, \omega)+S(z, x, \omega) .
$$

By using (2.3) and the fact that $\partial B_{r}(z)$ is disjoint from $C(\omega)$ we get

$$
\min _{z \in \partial B_{r}(x)} S(y, z, \omega)+S(z, x, \omega)>\min _{z \in \partial B_{r}(x)}(v(z)-v(y)+v(x)-v(z))=v(x)-v(y),
$$

as it was asserted.

Theorem 2.5. The definition of random Aubry set $\mathcal{A}(\omega)$ is well posed. Moreover, there is a critical subsolution which is weakly strict in $\mathbb{R}^{N} \backslash \mathcal{A}(\omega)$.

Under assumption (A), it has been provided in [10] a sort of dual characterization of the Aubry set as the minimal stationary closed random set for which there exists a critical admissible subsolution weakly strict in its complement. To prove the above theorem, we try to generalize this approach. We fix a sequence $\left(t_{n}\right)_{n}$ dense in $(0,+\infty)$ and, for each $v \in \mathcal{S}$, we define

$$
X_{v}^{t}(\omega)=\left\{x \in \mathbb{R}^{N}:\left(T_{t}^{-} v\right)(x, \omega)=v(x, \omega)\right\}, \quad t>0,
$$

and

$$
X_{v}(\omega)=\bigcup_{n \in \mathbb{N}} X_{v}^{t_{n}}(\omega)
$$

It is easily seen that $X_{v}^{t}(\omega)$ is a stationary closed random set. This yields that $X_{v}(\omega)$ is a stationary and graphmeasurable random set, but not closed in general.

We begin by showing a property enjoyed by such sets that will be relevant for our analysis. The following fact will be exploited in the proof and later on in the paper: when $v(\cdot, \omega)$ is a critical subsolution, the equality $\left(T_{t}^{-} v\right)(x, \omega)=$ $v(x, \omega)$ for some $t>0$ implies the existence of a point $y \in \mathbb{R}^{N}$ satisfying

$$
h_{t}(y, x, \omega)=v(x, \omega)-v(y, \omega)=S(y, x, \omega) .
$$

The last equality follows from Proposition 1.10 and (1.14) with $a=0$.

Proposition 2.6. Let $v \in \mathcal{S}$. Then there exists a set $\Omega_{v}$ of probability 1 such that, for every $\omega \in \Omega_{v}$ and $x_{0} \in X_{v}(\omega)$, any subtangent $\psi$ to $v(\cdot, \omega)$ at $x_{0}$ satisfies $H\left(x_{0}, D \psi\left(x_{0}\right), \omega\right) \geq 0$.

Proof. Let us fix $\omega$ in a set $\widehat{\Omega}$ of probability 1 such that $v(\cdot, \omega)$ is a critical subsolution and pick $x_{0} \in X_{v}(\omega)$. By definition of $X_{v}(\omega)$, there exist $t>0$ and $y \in \mathbb{R}^{N}$ with

$$
v\left(x_{0}, \omega\right)=v(y, \omega)+h_{t}\left(y, x_{0}, \omega\right)=v(y, \omega)+S\left(y, x_{0}, \omega\right) .
$$


This implies that the function $\bar{v}(\cdot):=S(y, \cdot, \omega)+v(y, \omega)$ touches $v(\cdot, \omega)$ from above at $x_{0}$, so any subtangent to $v(\cdot, \omega)$ at $x_{0}$ is also a subtangent to $\bar{v}$ at that point.

If $y \neq x_{0}$, the assertion follows since $\bar{v}$ is a critical solution in $\mathbb{R}^{N} \backslash\{y\}$. If instead $y=x_{0}$, then the we deduce from (2.7) the equality $h_{t}\left(x_{0}, x_{0}, \omega\right)=S\left(x_{0}, x_{0}, \omega\right)=0$. This means that there exists a closed curve $\gamma$ parameterized in the interval $[0, t]$ and with base point at $x_{0}$ along which the action of $L(\cdot, \cdot, \omega)$ is zero. By moving along $\gamma k$-times we infer that $h_{k t}\left(x_{0}, x_{0}, \omega\right)=0$ for any $k \in \mathbb{N}$. This, in turn, implies

$$
\liminf _{s \rightarrow+\infty} h_{s}\left(x_{0}, x_{0}, \omega\right)=0,
$$

and this relation is possible only when $c_{f}=0$ and $x_{0} \in \mathcal{A}_{f}(\omega)$. In this instance $\bar{v}$ is a critical solution on the whole $\mathbb{R}^{N}$, see [16] (or [14] for a proof in the case of a convex Hamiltonian just continuous). This concludes the proof.

Given $v \in \mathcal{S}$, we set

$$
\hat{v}(x, \omega):=v(x, \omega)-v(0, \omega) \text { for every }(x, \omega) \in \mathbb{R}^{N} \times \Omega .
$$

The set made up by critical subsolutions obtained in this way, namely

$$
\widehat{\mathcal{S}}:=\{\hat{v} \in \mathcal{S}: \hat{v}(0, \omega)=0 \text { for every } \omega\}
$$

is a subspace of $L^{0}\left(\Omega, \mathrm{C}\left(\mathbb{R}^{N}\right)\right)$, in particular it is separable with respect to the Ky Fan metric by Theorem 1.2. There thus exists a sequence of Lipschitz random functions $\left(v_{n}\right)_{n}$ dense in $\widehat{S}$ with respect to convergence in probability, which implies, in view of Theorem 1.1, that it is also dense for the almost sure convergence in $\mathrm{C}\left(\mathbb{R}^{N}\right)$. We set

$$
w(x, \omega)=\sum_{n=1}^{+\infty} \frac{1}{2^{n}} v_{n}(x, \omega) \quad \text { for every }(x, \omega) \in \mathbb{R}^{N} \times \Omega .
$$

The next result illustrates the first crucial property enjoyed by $w$.

Proposition 2.7. For every $v \in \mathcal{S}$, there exists a set $\Omega_{v}$ of probability 1 such that

$$
X_{w}^{t_{k}}(\omega) \subseteq X_{v}^{t_{k}}(\omega) \text { for every } \omega \in \Omega_{v} \text { and } k \in \mathbb{N} .
$$

In particular, there exists a set $\Omega_{v}$ of probability 1 such that

$$
X_{w}(\omega) \subseteq X_{v}(\omega) \text { for every } \omega \in \Omega_{v} .
$$

Proof. It is enough to prove the first assertion, for the second trivially follows from this. Let us fix $t \in\left\{t_{k}: k \in \mathbb{N}\right\}$. The proof is divided in two parts. First we prove the assertion for any element of the sequence $\left(v_{n}\right)_{n}$, then, in the second step, we extend it, by density, to all random functions in $\widehat{\mathcal{S}}$. This is actually enough, for $X_{v}^{t}(\omega)=X_{\hat{v}}^{t}(\omega)$, for any $\omega$, whenever $v$ and $\hat{v}$ are in the relation given by (2.8). Let us set

$$
\widehat{\Omega}:=\left\{\omega \in \Omega: v_{n}(\cdot, \omega) \text { is a critical subsolution for every } n \in \mathbb{N}\right\},
$$

and pick $\omega \in \widehat{\Omega}$ and $x \in X_{w}^{t}(\omega)$. Then $\left(T_{t}^{-} w\right)(x, \omega)=w(x, \omega)$. By the very definition of $T_{t}^{-}$

$$
\left(T_{t}^{-} w\right)(x, \omega) \geq \sum_{n=1}^{+\infty} \frac{1}{2^{n}}\left(T_{t}^{-} v_{n}\right)(x, \omega),
$$

and, combining this information with the monotonic character of the action of the negative Lax-Oleinik semigroup on critical subsolutions pointed out in Proposition 1.15, we get

$$
\left(T_{t}^{-} w\right)(x, \omega) \geq \sum_{n=1}^{+\infty} \frac{1}{2^{n}}\left(T_{t}^{-} v_{n}\right)(x, \omega) \geq \sum_{n=1}^{+\infty} \frac{1}{2^{n}} v_{n}(x, \omega)=w(x, \omega),
$$

discovering in the end that all the inequalities in the above formula have actually to be equalities. In particular we infer

$$
\left(T_{t}^{-} v_{n}\right)(x, \omega)=v_{n}(x, \omega) \quad \text { for every } n \in \mathbb{N},
$$


which proves that $X_{w}^{t}(\omega) \subseteq X_{v_{n}}^{t}(\omega)$ for every $\omega \in \widehat{\Omega}$ and $n \in \mathbb{N}$. Now let $v \in \widehat{\mathcal{S}}$. Then there exist a subsequence $\left(v_{n_{i}}\right)_{i}$ such that

$$
v_{n_{i}}(\cdot, \omega) \rightrightarrows v(\cdot, \omega) \quad \text { as } i \rightarrow+\infty
$$

for every $\omega$ in a set $\Omega_{v} \subseteq \widehat{\Omega}$ of probability 1 . Let $\omega \in \Omega_{v}$ and a pick a point $x \in X_{w}^{t}(\omega)$. In force of the first part of the proof, we have that (2.10) holds, and this readily implies $T_{t}^{-} v(x, \omega)=v(x, \omega)$ in view of (2.11). This shows that $x \in X_{v}^{t}(\omega)$, as desired.

In the next proposition we show that the action of the negative Lax-Oleinik semigroup does not affect the values of $w$ on $X_{w}(\omega)$ not only for some positive $t$, as required in the original definition of such a set, see (2.5), but actually for all $t>0$, at least a.s. in $\omega$.

Proposition 2.8. The following holds:

$$
X_{w}(\omega)=\left\{x \in \mathbb{R}^{N}:\left(T_{t}^{-} w\right)(x, \omega)=w(x, \omega) \text { for every } t>0\right\} \quad \text { a.s. in } \omega .
$$

Proof. Let $\widehat{\Omega}$ be a set of probability 1 such that $w(\cdot, \omega)$ is a critical subsolution. According to the monotonicity property stated in Proposition 1.14, the following implication holds for every $\omega \in \widehat{\Omega}$ :

$$
\left(T_{t}^{-} w\right)(x, \omega)=w(x, \omega) \quad \Rightarrow \quad T_{s}^{-} w(x, \omega)=w(x, \omega) \quad \text { for } s \in[0, t] .
$$

To prove the assertion, it will be enough to show that, for every $\omega$ in a set of probability 1 ,

$$
\sup \left\{t>0: T_{t}^{-} w(x, \omega)=w(x, \omega)\right\}=+\infty \quad \text { for every } x \in X_{w}(\omega) .
$$

For each $n \in \mathbb{N}$, set $w_{n}:=T_{t_{n}}^{-} w$, where $\left(t_{n}\right)_{n}$ is the dense sequence in $(0,+\infty)$ previously chosen in the definition of $X_{w}(\omega)$, see (2.5). According to Proposition 2.7, there is a set $\Omega^{\prime} \subseteq \widehat{\Omega}$ of probability 1 with

$$
X_{w}^{t_{n}}(\omega) \subset X_{w_{n}}^{t_{n}}(\omega) \text { for any } n \in \mathbb{N} \text { and } \omega \in \Omega^{\prime} .
$$

Fix $\omega \in \widehat{\Omega}, x \in X_{w}(\omega)$, and assume for purposes of contradiction

$$
\max \left\{t>0: T_{t}^{-} w(x, \omega)=w(x, \omega)\right\}=: s<\infty,
$$

then select $t_{k}$ with

$$
t_{k}<s<2 t_{k} .
$$

By (2.12)

$$
\left(T_{t_{k}}^{-} w\right)(x, \omega)=w(x, \omega),
$$

or, in other terms, $x \in X_{w}^{t_{k}}(\omega)$. In view of (2.13) we get $x \in X_{w_{k}}^{t_{k}}(\omega)$, i.e.

$$
T_{t_{k}}^{-}\left(T_{t_{k}}^{-} w\right)(x, \omega)=\left(T_{t_{k}}^{-} w\right)(x, \omega) .
$$

Combining it with (2.15), we get

$$
\left(T_{2 t_{k}}^{-} w\right)(x, \omega)=w(x, \omega),
$$

which is in contrast with (2.14) and the maximality property of $s$.

We go on gathering some more information on $X_{w}$.

Proposition 2.9. Let $c_{f}=0$. There exists a set $\widehat{\Omega}$ of probability 1 such that

$$
\mathcal{A}_{f}(\omega) \subseteq X_{w}(\omega) \text { for every } \omega \in \widehat{\Omega} .
$$


Proof. Let $\widehat{\Omega}$ be a set of probability 1 such that $w(\cdot, \omega)$ is a critical subsolution. Then, exploiting Proposition 1.14 and the very definition of $T_{t}^{-} w$, we have, for every $t>0$ and $x \in \mathbb{R}^{N}$,

$$
w(x, \omega) \leq\left(T_{t}^{-} w\right)(x, \omega) \leq \liminf _{t \rightarrow+\infty} w(x, \omega)+h_{t}(x, x, \omega) .
$$

When $c_{f}=0$ and $x \in \mathcal{A}_{f}(\omega)$

$$
\liminf _{t \rightarrow+\infty} h_{t}(x, x, \omega)=0,
$$

so that, combining (2.16) and (2.17), we get in the end $w(x, \omega)=\left(T_{t}^{-} w\right)(x, \omega)$.

We pause our analysis on $w$ to derive a general characterization of weakly strict subsolutions in terms of strict monotonicity of the action of the negative Lax-Oleinik semigroup.

Lemma 2.10. Let $w \in \mathcal{S}$ and $C(\omega)$ be a stationary closed random set. If $c_{f}=0$, we additionally assume that $\mathcal{A}_{f}(\omega) \subseteq$ $C(\omega)$ a.s. in $\omega$. Then $w$ is weakly strict in $\mathbb{R}^{N} \backslash C(\omega)$ if and only if there exists a set $\widehat{\Omega}$ of probability 1 such that

$$
\left(T_{t}^{-} w\right)(y, \omega)>w(y, \omega) \quad \text { for any } y \in \mathbb{R}^{N} \backslash C(\omega), \omega \in \widehat{\Omega} \text { and } t>0 .
$$

Proof. Let $\widehat{\Omega}$ be a set of probability 1 made up of elements $\omega \in \Omega$ for which $w(\cdot, \omega)$ is a critical subsolution and enjoys (2.3). Throughout the proof, $\omega$ will denote a fixed element of $\widehat{\Omega}$. Were (2.18) not true, we should have by Proposition 1.14

$$
\left(T_{t}^{-} w\right)(y, \omega)=w(y, \omega)
$$

for some $t>0$ and $y \notin C(\omega)$, hence, by (2.6),

$$
w(y, \omega)-w(z, \omega)=S(z, y, \omega) \text { for some } z \in \mathbb{R}^{N},
$$

in contrast with the fact that $w(\cdot, \omega)$ is weakly strict and $y \notin C(\omega)$.

The converse implication will be also proved by contradiction. Assume that (2.19) holds for some $y, z$ not belonging to $C(\omega)$. Exploiting that $S(\cdot, \cdot, \omega)$ is a geodesic semidistance, we can assume, up to moving $y$, that there is a curve $\gamma:[0,1] \rightarrow \mathbb{R}^{N}$ joining $z$ to $y$ and with support disjoint from $C(\omega)$ such that

$$
w(y, \omega)-w(z, \omega)=\int_{0}^{1} \sigma(\gamma, \dot{\gamma}, \omega) \mathrm{d} s .
$$

Since the support of $\gamma$ is also by assumption disjoint from $\mathcal{A}_{f}(\omega)$ in the case that $c_{f}=0$, we can determine a reparametrization $\xi:[0, t] \rightarrow \mathbb{R}^{N}$ of $\gamma$, for some $t>0$, with

$$
\sigma(\xi(s), \dot{\xi}(s), \omega)=L(\xi(s), \dot{\xi}(s), \omega) \text { for a.e. } s \in[0, t],
$$

see $[9,13]$. We deduce

$$
w(y, \omega)=w(z, \omega)+\int_{0}^{t} L(\gamma, \dot{\gamma}, \omega) \mathrm{d} s \geq\left(T_{t}^{-} w\right)(y, \omega),
$$

but since the opposite inequality comes from Proposition 1.14, the above formula must actually hold with equality, yielding a contradiction.

We are now in position to give the

Proof of Theorem 2.5. Let $w$ be defined via (2.9) and set

$$
C(\omega)=\bigcap_{n \in \mathbb{N}}\left\{x \in \mathbb{R}^{N}:\left(T_{s_{n}}^{-} w\right)(x, \omega)=w(x, \omega)\right\} \quad \text { for every } \omega,
$$


where $\left(s_{n}\right)_{n}$ is any fixed dense sequence in $(0,+\infty)$ (for instance, we could take the one previously chosen in the definition of $X_{w}(\omega)$, see (2.5)). It is easily seen that $C(\omega)$ is stationary closed random set. Moreover, by the monotonicity property stated in Proposition 1.14 and by Proposition 2.8,

$$
C(\omega)=X_{w}(\omega) \quad \text { a.s. in } \omega .
$$

We claim that $w$ is weakly strict in $\mathbb{R}^{N} \backslash C(\omega)$ and that $C(\omega)$ is the Aubry set.

Let us start with the weakly strict character of $w$ in $\mathbb{R}^{N} \backslash C(\omega)$. In view of (2.20) and of Proposition 2.9, for every $\omega$ in a set of probability 1 we have

$$
\left(T_{t}^{-} w\right)(y, \omega)>w(y, \omega) \text { for every } y \in \mathbb{R}^{N} \backslash C(\omega) \text { and } t>0
$$

and $\mathcal{A}_{f}(\omega) \subseteq C(\omega)$ if $c_{f}=0$. By Lemma 2.10, we conclude that $w$ is weakly strict in $\mathbb{R}^{N} \backslash C(\omega)$.

Let us now show that $C(\omega)$ is the Aubry set, i.e. it is the maximal stationary closed random set satisfying item $(i)$ in Definition 2.2 . We start by proving the maximality property. Let $\widetilde{C}(\omega)$ be a nonempty stationary closed random set satisfying item $(i)$ in Definition 2.2 and set

$$
u(x, \omega):=\inf \{w(y, \omega)+S(y, x, \omega): y \in \widetilde{C}(\omega)\}, \quad x \in \mathbb{R}^{N} .
$$

Since $u$ is an admissible critical solution, we infer from Proposition 1.14 that the following equality holds for every $\omega$ in a set of probability 1 :

$$
T_{t}^{-} u(\cdot, \omega) \equiv u(\cdot, \omega) \quad \text { on } \mathbb{R}^{N} \text { for every } t>0 .
$$

Take $\omega \in \Omega$ such that $w(\cdot, \omega)$ is a critical subsolution, $\widetilde{C}(\omega) \neq \emptyset$ and $(2.22)$ holds. We know that $w(\cdot, \omega) \leq u(\cdot, \omega)$ in $\mathbb{R}^{N}$ and $w(\cdot, \omega)=u(\cdot, \omega)$ in $\widetilde{C}(\omega)$. By the monotone character of the operator $T_{t}^{-}$, for every $x \in \widetilde{C}(\omega)$ we deduce

$$
w(x, \omega) \leq\left(T_{t}^{-} w\right)(x, \omega) \leq\left(T_{t}^{-} u\right)(x, \omega)=w(x, \omega) \text { for every } t>0 .
$$

Hence all the inequalities in the above formula are indeed equalities, yielding $\widetilde{C}(\omega) \subseteq C(\omega)$ a.s. in $\omega$.

In particular, we derive that $\mathcal{A}(\omega)=\emptyset$ when $C(\omega)$ is almost surely empty, since the above argument implies, in this instance, that stationary and nonempty closed random sets satisfying $(i)$ in Definition 2.2 do not exist.

Let us then assume that $C(\omega) \neq \varnothing$ a.s. in $\omega$ and let us check that it satisfies $(i)$ in Definition 2.2. Pick a critical subsolution $g \in S$ and let $u$ be the admissible critical subsolution defined through (2.1). To prove that $u(\cdot, \omega)$ is an almost sure critical solution on $\mathbb{R}^{N}$, we only have to check, in view of (2.2), that the supersolution test is satisfied on $C(\omega)$ a.s. in $\omega$. But this follows in view of (2.20) and of Propositions 2.7 and 2.6 with $v:=u$.

Remark 2.11. Since $v$ is a subsolution of $H=a$ if and only if $-v$ is a subsolution of $\check{H}=a$, then $H$ and $\check{H}$ have the same critical value. Moreover, if $v$ is a critical subsolution for $H$ which is weakly strict outside some stationary closed random set $C(\omega)$, then $-v$ is critical for $\check{H}$ and weakly strict outside $C(\omega)$, see Proposition 5.10 in [10] for more details. In view of Theorem 2.5 and Remark 2.13, this implies that $H$ and $\breve{H}$ have the same Aubry set.

We finally record for later use that, as a consequence of Theorem 2.5, we are able to extend Theorem 5.9 in [10], employing the same argument used there, as follows:

Theorem 2.12. Assume that $\mathcal{A}(\omega) \neq \emptyset$ a.s. in $\omega$. Then there exists a set $\widehat{\Omega}$ of probability 1 such that for any $\omega \in \widehat{\Omega}$ and any $x \in \mathcal{A}(\omega)$ we can find a curve $\eta_{x}: \mathbb{R} \rightarrow \mathcal{A}(\omega)$ (depending on $\omega$ ) with $\eta_{x}(0)=x$ satisfying the following properties:

(i) for every $a<b$ in $\mathbb{R}$

$$
S\left(\eta_{x}(a), \eta_{x}(b), \omega\right)=\int_{a}^{b} L\left(\eta_{x}, \dot{\eta}_{x}, \omega\right) \mathrm{d} s
$$


(ii) for every $v \in \mathcal{S}$ there exists a set $\Omega_{v}$ of probability 1 such that for every $\omega \in \Omega_{v}$

$$
\int_{a}^{b} L\left(\eta_{x}, \dot{\eta}_{x}, \omega\right) \mathrm{d} s=v\left(\eta_{x}(b), \omega\right)-v\left(\eta_{x}(a), \omega\right) \quad \text { for every } a<b \text { in } \mathbb{R} .
$$

If condition (A) holds, we furthermore have $\lim _{t \rightarrow \pm \infty}\left|\eta_{x}(t)\right|=+\infty$.

Remark 2.13. From Theorems 2.5 and 2.12 we deduce that $\mathcal{A}(\omega)$ is the minimal stationary closed random set for which there exists a critical admissible subsolution which is weakly strict in its complement.

\subsection{Strict critical subsolutions}

The purpose of this section is to reinforce Theorem 2.5 showing the existence of a critical subsolution enjoying the property of being strict outside the Aubry set in a stronger and more classical sense.

Definition 2.14. Let $C(\omega)$ be a stationary closed random set. A critical subsolution $v \in \mathcal{S}$ is said to be strict in $\mathbb{R}^{N} \backslash C(\omega)$ if the following property holds a.s. in $\omega$ :

for every open set $U$ compactly contained in $\mathbb{R}^{N} \backslash C(\omega)$ there exists $\delta>0$ such that

$$
H(x, D v(x, \omega), \omega) \leq-\delta \text { for a.e. } x \in U .
$$

We will say that $v$ is (weakly) strict, with no further specification, to mean that it is (weakly) strict in $\mathbb{R}^{N} \backslash \mathcal{A}(\omega)$.

Next lemma makes precise that the previous notion is actually a strengthening of that of weakly strict subsolution. It is a purely deterministic result, where $\omega$ plays just the role of a parameter, and so is omitted for notational simplicity.

Lemma 2.15. Let $v$ be a critical subsolution satisfying (2.23) in some open subset $U$ of $\mathbb{R}^{N}$ and for some $\delta>0$. Then

$$
v(x)-v(y)<S(y, x) \text { for every } x \in U \text { and } y \neq x .
$$

Proof. Since $S$ is a geodesic-type semidistance, it will be enough to check (2.24) for every point $x \in U$ and $y \in$ $\partial B_{r}(x)$, where $B_{r}(x)$ is any open ball compactly contained in $U$.

Let $\rho=\rho(0,-\delta)>0$ be chosen according to (1.12) and choose a curve $\gamma$ joining $y$ to $x$ such that

$$
\frac{\rho r}{2}+S(y, x)>\int_{0}^{1} \sigma(\gamma, \dot{\gamma}) \mathrm{d} s .
$$

Let us set $\tau:=\sup \left\{t \in[0,1]: \gamma(t) \in \mathbb{R}^{N} \backslash B_{r}(x)\right\}$ and $z=\gamma(\tau)$. By taking into account (1.12) and the fact that the critical subsolution $v$ satisfies $(2.23)$ in $B_{r}(x)$, we get:

$$
\begin{aligned}
\int_{0}^{1} \sigma(\gamma, \dot{\gamma}) \mathrm{d} s & =\int_{0}^{\tau} \sigma(\gamma, \dot{\gamma}) \mathrm{d} s+\int_{\tau}^{1} \sigma(\gamma, \dot{\gamma}) \mathrm{d} s \\
& \geq v(z)-v(y)+v(x)-v(z)+\rho \int_{\tau}^{1}|\dot{\gamma}(s)| \mathrm{d} s .
\end{aligned}
$$

Hence by (2.25)

$$
S(y, x) \geq v(x)-v(y)+\frac{\rho r}{2}>v(x)-v(y) .
$$

The converse implication does not hold. More generally, the inequality

$$
v(x)-v(y)<S_{a}(y, x) \text { for every } x, y \text { in an open set } U \subseteq \mathbb{R}^{N}
$$


does not imply ess $\sup _{x \in U} H(x, D v(x))<a$. For instance, the antiderivative of a function vanishing on an open and dense subset of $\mathbb{R}$ of small measure and equal to 1 in the complement is a weakly strict subsolution of the 1 -dimensional Eikonal equation $\left|u^{\prime}\right|=1$ in $\mathbb{R}$, but it is not strict in $\mathbb{R}$.

The statement of the main theorem reads as follows:

Theorem 2.16. Let $H$ satisfy (H1)-(H4). Then there exists a strict critical subsolution in $\mathcal{S}$. More precisely, for every weakly strict critical subsolution $w \in \mathcal{S}$ and every $\varepsilon>0$, there exists a strict critical subsolution $w_{\varepsilon} \in \mathcal{S}$ such that

(i) $\left\|w_{\varepsilon}(\cdot, \omega)-w(\cdot, \omega)\right\|_{\infty}<\varepsilon$ for every $\omega \in \Omega$;

(ii) $w_{\varepsilon}(\cdot, \omega)=w(\cdot, \omega)$ on $\mathcal{A}(\omega)$ a.s. in $\omega$.

It, in particular, implies the existence of a critical admissible subsolution, strict on the whole $\mathbb{R}^{N}$, when the random Aubry set is almost surely empty.

As a consequence, we also get:

Corollary 2.17. Let $H$ satisfy (H1)-(H4). The set of admissible, strict critical subsolutions is dense in $\mathcal{S}$ with respect to the Ky Fan metric on $L^{0}\left(\Omega ; \mathrm{C}\left(\mathbb{R}^{N}\right)\right)$.

Proof. According to Theorems 2.5 and 2.16, there exists a strict critical subsolution in $\mathcal{S}$, say it $v$. Now pick $u \in \mathcal{S}$ and set

$$
v_{n}(x, \omega)=\frac{1}{n} v(x, \omega)+\left(1-\frac{1}{n}\right) u(x, \omega) \quad(x, \omega) \in \mathbb{R}^{N} \times \Omega
$$

for every $n \in \mathbb{N}$. By convexity of the Hamiltonian, $v_{n}$ are strict critical subsolutions belonging to $\mathcal{S}$. Moreover

$$
v_{n}(\cdot, \omega) \rightrightarrows u(\cdot, \omega) \quad \text { for every } \omega \in \Omega,
$$

meaning that $d\left(v_{n}(\cdot, \omega), u(\cdot, \omega)\right) \rightarrow 0$ for every $\omega \in \Omega$. Since almost sure convergence implies convergence in probability, we get that $v_{n}$ converge to $u$ with respect to the Ky Fan metric in $L^{0}\left(\Omega ; \mathrm{C}\left(\mathbb{R}^{N}\right)\right)$ in view of Theorem 1.2.

To pass from the existence of a weakly strict admissible subsolution to that of a strict one, we make use of two, in a sense complementary, crucial properties of the Lax-Oleinik semigroups that will be proved below. The first is the invariance of the values of any critical subsolution on the random Aubry set under the action of $T_{t}^{-}$and $T_{t}^{+}$, the second instead the strict monotonicity of $T_{t}^{-}$, when applied to a weakly strict critical subsolution, outside such set, at least for small times.

Proposition 2.18. Let $w \in \mathcal{S}$. Then the following property holds a.s. in $\omega$ :

$$
T_{t}^{-} w(x, \omega)=T_{t}^{+} w(x, \omega)=w(x, \omega) \quad \text { for any } x \in \mathcal{A}(\omega) \text { and } t>0 .
$$

Proof. We assume that Aubry set is a.s. nonempty otherwise the statement is void. We take $\omega$ such that $\mathcal{A}(\omega) \neq \emptyset$, $w(\cdot, \omega)$ is a subsolution of the corresponding critical Hamilton-Jacobi equation and assertion (ii) of Theorem 2.12 holds for $v:=w$. Pick a point $x \in \mathcal{A}(\omega)$ and a time $t>0$. According to Proposition 1.14,

$$
T_{t}^{-} w(x, \omega) \geq w(x, \omega) .
$$

Now, let $\eta_{x}: \mathbb{R} \rightarrow \mathbb{R}^{N}$ be the curve chosen according to Theorem 2.12. Then

$$
w(x, \omega)=w\left(\eta_{x}(-t), \omega\right)+\int_{-t}^{0} L\left(\eta_{x}, \dot{\eta}_{x}, \omega\right) \mathrm{d} s \geq\left(T_{t}^{-} w\right)(x, \omega),
$$

yielding equality. The assertion for $T_{t}^{+} w$ can be proved analogously in view of Remarks 1.11 and 2.11. 
Proposition 2.19. Let $w \in \mathcal{S}$ be weakly strict. Then we can determine a set $\Omega_{w}$ of probability 1 such that for every $\omega \in \Omega_{w}$ and $x \in \mathbb{R}^{N} \backslash \mathcal{A}(\omega)$ there exists $t_{x}=t_{x}(\omega) \in(0,+\infty]$ such that the function

$$
t \mapsto v(t, x, \omega):=\left(T_{t}^{-} w\right)(x, \omega)
$$

is strictly increasing in $\left[0, t_{x}\right)$ and constant for $t \geq t_{x}$.

Proof. Let $\Omega_{w}$ be a set of probability 1 made up by elements $\omega$ for which $w(\cdot, \omega)$ is a weakly strict critical subsolution. Fix $\omega \in \Omega_{w}$ and $x \in \mathbb{R}^{N} \backslash \mathcal{A}(\omega)$. Let us define $J$ as the set of times $t>0$ satisfying the following property:

$$
\left(T_{t}^{-} w\right)(x, \omega)=w(y, \omega)+h_{t}(y, x, \omega) \text { for some } y \in \mathcal{A}(\omega) .
$$

Let us set $t_{x}:=\inf J$, where we agree that $t_{x}=+\infty$ if $J$ is empty. From Proposition 1.12, which bounds the distance of the point $y$ in (2.26) from $x$, and the fact that $\mathcal{A}(\omega)$ is closed we infer that $J$ is closed and that $t_{x}>0$.

Let us prove that $t \mapsto\left(T_{t}^{-} w\right)(x, \omega)$ is strictly increasing in $\left[0, t_{x}\right)$. Take $s>t$ in $\left[0, t_{x}\right)$, then

$$
\left(T_{s}^{-} w\right)(x, \omega)=\left(T_{s-t}^{-} w\right)(y, \omega)+h_{t}(y, x, \omega) \text { for some } y \in \mathbb{R}^{N} .
$$

If $y \in \mathcal{A}(\omega)$, from Proposition 2.18 we deduce

$$
\left(T_{s}^{-} w\right)(x, \omega)=w(y, \omega)+h_{t}(y, x, \omega)>\left(T_{t}^{-} w\right)(x, \omega),
$$

were the strict inequality comes from the fact that $t \notin J$. If instead $y \notin \mathcal{A}(\omega)$, we invoke Lemma 2.10, which holds true for the $\omega$ we are working with, to get from $(2.27)$

$$
\left(T_{s}^{-} w\right)(x, \omega)>w(y, \omega)+h_{t}(y, x, \omega) \geq\left(T_{t}^{-} w\right)(x, \omega) .
$$

Let us now prove that $t \mapsto\left(T_{t}^{-} w\right)(x, \omega)$ is constant in $\left[t_{x},+\infty\right)$ when $t_{x}<+\infty$. Let $y \in \mathcal{A}(\omega)$ be a point satisfying (2.26) with $t_{x}$ in place of $t$. We invoke Proposition 2.18 to get for $t>t_{x}$

$$
\left(T_{t}^{-} w\right)(x, \omega) \leq\left(T_{t-t_{x}}^{-} w\right)(y, \omega)+h_{t_{x}}(y, x, \omega)=w(y, \omega)+h_{t_{x}}(y, x, \omega)=\left(T_{t_{x}}^{-} w\right)(x, \omega) .
$$

By monotonicity properties of Lax-Oleinik semigroup pointed out in Proposition 1.14, we get the assertion.

To proceed in our analysis, we need two technical lemmata about locally Lipschitz functions and related Clarke's generalized gradients. In what follows, we denote by $\pi_{1}, \pi_{2}$ the maps defined as $\pi_{1}\left(p_{t}, p_{x}\right)=p_{t}, \pi_{2}\left(p_{t}, p_{x}\right)=p_{x}$ for every $\left(p_{t}, p_{x}\right) \in \mathbb{R} \times \mathbb{R}^{N}$.

Lemma 2.20. Let $v(t, x)$ be a locally Lipschitz function in $(0,+\infty) \times \mathbb{R}^{N}$ such that, for every bounded open subset $U$ of $\mathbb{R}^{N}$, the functions

$$
\{v(\cdot, x): x \in U\} \text { are locally equi-semiconcave (resp. equi-semiconvex) in }(0,+\infty) \text {. }
$$

Then, for every $\left(t_{0}, x_{0}\right) \in(0,+\infty) \times \mathbb{R}^{N}$,

$$
\pi_{1}\left(\partial^{c} v\left(t_{0}, x_{0}\right)\right)=\partial_{t}^{c} v\left(t_{0}, x_{0}\right), \quad \pi_{2}\left(\partial^{c} v\left(t_{0}, x_{0}\right)\right) \supseteq \partial_{x}^{c} v\left(t_{0}, x_{0}\right) .
$$

In particular, the map $(t, x) \mapsto \partial_{t}^{c} v(t, x)$ is upper semicontinuous in $(0,+\infty) \times \mathbb{R}^{N}$.

Proof. The fact that

$$
\pi_{1}\left(\partial^{c} v\left(t_{0}, x_{0}\right)\right) \supseteq \partial_{t}^{c} v\left(t_{0}, x_{0}\right), \quad \pi_{2}\left(\partial^{c} v\left(t_{0}, x_{0}\right)\right) \supseteq \partial_{x}^{c} v\left(t_{0}, x_{0}\right)
$$

for every $\left(t_{0}, x_{0}\right) \in(0,+\infty) \times \mathbb{R}^{N}$ follows from Proposition 2.3.16 of [6]. To prove that $\pi_{1}\left(\partial^{c} v\left(t_{0}, x_{0}\right)\right)=\partial_{t}^{c} v\left(t_{0}, x_{0}\right)$, it will be enough to show that

$$
\pi_{1}\left(\partial^{*} v\left(t_{0}, x_{0}\right)\right) \subseteq \partial_{t}^{c} v\left(t_{0}, x_{0}\right) .
$$

Let $p_{t_{0}} \in \pi_{1}\left(\partial^{*} v\left(t_{0}, x_{0}\right)\right)$ and let $\left(t_{n}, x_{n}\right)$ be a sequence of differentiability points for $v$ converging to $\left(t_{0}, x_{0}\right)$ such that $\partial_{t} v\left(t_{n}, x_{n}\right)=: p_{t_{n}} \rightarrow p_{t_{0}}$ as $n \rightarrow+\infty$. The functions

$$
\phi_{n}(t):=w\left(t-t_{0}+t_{n}, x_{n}\right)
$$


are locally equi-semiconcave (resp. equi-semiconvex) in $(0,+\infty)$ and

$$
\phi_{n} \rightrightarrows v\left(\cdot, x_{0}\right) \quad \text { in }(0,+\infty) .
$$

Moreover $\phi_{n}$ are differentiable at $t_{0}$ and $\phi_{n}^{\prime}\left(t_{0}\right)=p_{t_{n}}$, in particular $p_{t_{n}}$ belongs to the superdifferential (resp. subdifferential) of $\phi_{n}$ at $t_{0}$ by semiconcavity (resp. semiconvexity). This property passes to the limit since the functions $\phi_{n}$ are locally equi-semiconcave (resp. equi-semiconvex), hence $p_{t_{0}}$ belongs to the superdifferential (resp. subdifferential) of the function $v\left(\cdot, x_{0}\right)$ at $t_{0}$, that is to $\partial_{t}^{c} v\left(t_{0}, x_{0}\right)$.

Since the map $(t, x) \mapsto \partial^{c} v(t, x)$ is upper semicontinuous in $(0,+\infty) \times \mathbb{R}^{N}$, so is the map $(t, x) \mapsto \pi_{1}\left(\partial^{c} v(t, x)\right)$, from which we get the last assertion in view of the identity just established.

Lemma 2.21. Let $v(t, x)$ be a locally Lipschitz function in $(0,+\infty) \times \mathbb{R}^{N}$ and assume there is a bounded open subset $U$ of $\mathbb{R}^{N}$ such that

(i) the functions $\{v(\cdot, x): x \in U\}$ are locally equi-semiconcave (resp. equi-semiconvex) in $(0,+\infty)$;

(ii) for every $x \in U$ the map

$$
t \mapsto v(t, x) \quad \text { is strictly increasing in }\left[0, t_{x}\right)
$$

for some $t_{x} \in(0,+\infty]$.

Then, for every $x \in U$, the set

$$
I_{x}:=\left\{t>0: \min \left\{p_{t}: p_{t} \in \partial_{t}^{c} v(t, x)\right\}>0\right\}
$$

is open and dense in $\left(0, t_{x}\right)$, in particular it is nonempty.

Proof. Let us fix $x \in U$ and set

$$
f(t)=\min \left\{p_{t}: p_{t} \in \partial_{t}^{c} v(t, x)\right\} \quad \text { for every } t>0 .
$$

Thanks to Lemma 2.20, the set-valued map $t \mapsto \partial_{t}^{c} v(t, x)$ is upper semicontinuous. Consequently, the function $f$ is lower semicontinuous, so that

$$
I_{x}=\{t>0: f(t)>0\}
$$

is open. Let us prove that it is dense in $\left(0, t_{x}\right)$. This is indeed a consequence of the strict monotonicity of $t \mapsto v(t, x)$ in $\left[0, t_{x}\right)$. Because of it, in fact, in any subinterval of $\left(0, t_{x}\right)$ we find differentiability points of $v(\cdot, x)$ with positive derivative. Since any such point, say $t_{0}$, is of strict differentiability due to the semiconcavity (resp. semiconvexity) of $v(\cdot, x)$, the corresponding derivative is also the unique generalized gradient, which shows that $t_{0} \in I_{x}$ and proves the statement.

Our strategy for proving Theorem 2.16 is structured in two parts: we first show the result under the additional assumption that the $H$ is strictly convex and then generalize it to Hamiltonians solely convex by means of a regularization in time of the action of the negative Lax-Oleinik semigroup via $t$-partial sup convolutions.

Proof of Theorem 2.16 for $\boldsymbol{H}$ strictly convex. The precise statement of our extra assumption is:

$\left(\mathrm{H} 2^{\prime}\right) H(x, \cdot, \omega)$ is strictly convex on $\mathbb{R}^{N}$ for every $(x, \omega) \in \mathbb{R}^{N} \times \Omega$.

Let $w \in \mathcal{S}$ be a weakly strict subsolution and let $\varepsilon>0$ be fixed. The main effect of $\left(\mathrm{H} 2^{\prime}\right)$ is that the function

$$
v(t, x, \omega):=\left(T_{t}^{-} w\right)(x, \omega), \quad(t, x, \omega) \in(0,+\infty) \times \mathbb{R}^{N} \times \Omega,
$$

is locally semiconcave in $(0,+\infty)$ with respect to $t$, see Lemma 2.11 in [14]. More precisely, for every open and bounded set $U \subset \mathbb{R}^{N}$ and every fixed $\omega \in \Omega$ the functions

$$
\{v(\cdot, x, \omega): x \in U\} \quad \text { are locally equi-semiconcave in }(0,+\infty) .
$$


Let $\kappa$ be the constant given by (1.8) with $a=0$ and let $R(\kappa)$ be chosen according to Proposition 1.12. Choose $\tau>0$ such that $\tau R(\kappa)<\varepsilon$ and let $\left(t_{n}\right)_{n}$ be a dense sequence in $(0, \tau)$. We define

$$
w_{\varepsilon}(x, \omega)=\sum_{n=1}^{+\infty} \frac{1}{2^{n}} v\left(t_{n}, x, \omega\right), \quad(x, \omega) \in \mathbb{R}^{N} \times \Omega .
$$

By Proposition 1.15, we get

$$
\left\|w_{\varepsilon}(\cdot, \omega)-w(\cdot, \omega)\right\|_{\infty} \leq \sum_{n=1}^{+\infty} \frac{1}{2^{n}}\left\|v\left(t_{n}, \cdot, \omega\right)-w(\cdot, \omega)\right\|_{\infty} \leq \tau R(\kappa)<\varepsilon
$$

for every $\omega \in \Omega$, showing in particular that $w_{\varepsilon}(\cdot, \omega)$ is finite-valued. As a convex combination of admissible critical subsolutions, see Proposition 1.15, a standard argument shows that $w_{\varepsilon}$ is an admissible subsolution as well. From Proposition 2.18 we also infer

$$
w_{\varepsilon}(\cdot, \omega)=w(\cdot, \omega) \quad \text { on } \mathcal{A}(\omega)
$$

a.s. in $\omega$. It is left to show that $w_{\varepsilon}$ is strict. To this purpose, let us fix $\omega$ in a set of probability 1 made up of elements for which the assertion of Proposition 2.19 holds true and the functions $v\left(t_{n}, \cdot, \omega\right)$ are critical subsolutions. Pick a point $y \in \mathbb{R}^{N} \backslash \mathcal{A}(\omega)$. According to Lemma 2.21 and by density of the sequence $\left(t_{n}\right)_{n}$ in $(0, \tau)$, there exist $i \in \mathbb{N}$ and $a>0$ such that

$$
\min \left\{p_{t}: p_{t} \in \partial_{t}^{c} v\left(t_{i}, y, \omega\right)\right\}>a .
$$

By upper semicontinuity of the set-valued map $x \mapsto \partial_{t}^{c} v\left(t_{i}, x, \omega\right)$, we infer that (2.31) keep holding in a ball $B_{r}(y)$. Now we exploit the fact that $v(\cdot, \cdot, \omega)$ is a (sub)solution of the time-dependent equation (1.16), in view of Proposition 1.13 , so

$$
p_{t}+H\left(x, p_{x}, \omega\right) \leq 0 \text { for every }\left(p_{t}, p_{x}\right) \in \partial^{c} v\left(t_{i}, x, \omega\right) \text { and } x \in B_{r}(y) .
$$

In view of Lemma 2.20 and of what remarked above, we get in particular

$$
H\left(x, D_{x} v\left(t_{i}, x, \omega\right), \omega\right)<-a<0 \quad \text { for a.e. } x \in B_{r}(y) .
$$

By the definition of $w_{\varepsilon}$ and the convexity of $H$, we conclude that

$$
\begin{aligned}
H\left(x, D w_{\varepsilon}(x, \omega), \omega\right) & \leq \frac{1}{2^{i}} H\left(x, D_{x} v\left(t_{i}, x, \omega\right), \omega\right) \\
& +\sum_{n \neq i} \frac{1}{2^{n}} H\left(x, D_{x} v\left(t_{n}, x, \omega\right), \omega\right) \leq-\frac{a}{2^{i}}<0
\end{aligned}
$$

for a.e. $x \in B_{r}(y)$. This actually shows that $w_{\varepsilon}$ is strict since $y$ was arbitrarily chosen in $\mathbb{R}^{N} \backslash \mathcal{A}(\omega)$.

Looking carefully at the above argument, we recognize that definition (2.28) can be interpreted as a convenient way to select a 1-parameter family $\{v(t, \cdot, \cdot)\}_{t>0}$ of elements in $\mathcal{S}$ in such a way that the following conditions are satisfied almost surely: $v(\cdot, \cdot, \omega)$ is a subsolution of the time-dependent equation (1.16); the function $t \mapsto v(t, x, \omega)$ is constant on $\mathcal{A}(\omega)$, and strictly increasing outside $\mathcal{A}(\omega)$ in a suitable neighborhood $\left[0, t_{x}\right)$ of $t=0$; the map $t \mapsto v(t, x, \omega)$ is locally semiconcave (or semiconvex) in $(0,+\infty)$, with a modulus that is locally uniform with respect to $x$.

In the general case of a Hamiltonian, not strictly convex, but just convex, the latter condition is apparently no longer fulfilled by the random variable given by (2.28). To get the proof of Theorem 2.16 in full generality, we modify the definition of $v$ by setting

$$
v(t, x, \omega)=\sup _{s \geq 0}\left\{\left(T_{s}^{-} w\right)(x, \omega)-\frac{1}{2 \delta}(s-t)^{2}\right\} .
$$

To complete our task it is then enough to check out that such a $v$ fulfills all the requirements listed above, and this is indeed the content of our next proposition. In this way $v$ can be actually used in formula (2.29) to provide a strict subsolution $w_{\varepsilon} \in \mathcal{S}$ almost surely satisfying (2.30), while for the inequality $\left\|w_{\varepsilon}-w\right\|_{\infty}<\varepsilon$ in the item (i) of the statement, it simply suffices to choose $\tau>0$ and $\delta>0$ small enough. 
Proposition 2.22. Let $v$ be defined via (2.32) for some $w \in \mathcal{S}$ and $\delta>0$. The following properties hold:

(i) $\|v(t, \cdot, \omega)-w(\cdot, \omega)\|_{\infty} \leq R(\kappa)(t+2 \delta R(\kappa))$ for every $t>0$ and $\omega \in \Omega$;

(ii) the function $v(\cdot, \cdot, \omega)$ is a Lipschitz subsolution in $(0,+\infty) \times \mathbb{R}^{N}$ of the time-dependent equation (1.16) for every $\omega \in \Omega$;

(iii) $v(t, \cdot, \cdot) \in \mathcal{S}$ for every $t>0$;

(iv) for every $\omega$ in a set of probability 1 the following property holds:

$$
v(t, \cdot, \omega)=w(\cdot, \omega) \quad \text { on } \mathcal{A}(\omega) \text { for every } t>0 ;
$$

(v) the functions $\left\{v(\cdot, x, \omega): x \in \mathbb{R}^{N}, \omega \in \Omega\right\}$ are equi-semiconvex in $(0,+\infty)$.

Moreover, if $w \in \mathcal{S}$ is weakly strict, then $v$ enjoys the assertion of Proposition 2.19.

Proof. By Proposition 1.12 we know that the function $(t, x) \mapsto\left(T_{t}^{-} w\right)(x, \omega)$ is $R(\kappa)$-Lipschitz continuous $[0,+\infty) \times \mathbb{R}^{N}$ for every $\omega \in \Omega$, in particular $\left(T_{t}^{-} w\right)(x, \omega)$ grows at most linearly in $t$. This implies that the supremum in (2.32) is attained. Let us denote by $Y(t, x, \omega)$ the set of maximizers of the right-hand side term of (2.32). Then

$$
|s-t| \leq 2 \delta R(\kappa) \quad \text { for every } s \in Y(t, x, \omega) .
$$

This follows from the fact that, for any such $s$, we have

$$
\frac{1}{2 \delta}(t-s)^{2} \leq\left(T_{s}^{-} w\right)(x, \omega)-\left(T_{t}^{-} w\right)(x, \omega) \leq R(\kappa)|t-s| .
$$

In particular,

$$
0 \leq v(t, x, \omega)-\left(T_{t}^{-} w\right)(x, \omega) \leq\left(T_{s}^{-} w\right)(x, \omega)-\left(T_{t}^{-} w\right)(x, \omega) \leq 2 \delta R(\kappa)^{2},
$$

and assertion (i) follows by Proposition 1.12-(ii).

Assertion (ii) is well known, see for instance [5]: the Lipschitz character of $v(\cdot, \cdot, \omega)$ comes from the fact that it is the supremum of equi-Lipschitz functions; the subsolution property is a consequence of the inclusion

$$
D^{+} v(t, x, \omega) \subseteq D^{+}\left(T_{s}^{-} w\right)(x, \omega) \quad \text { for every } s \in Y(t, x, \omega),
$$

together with the fact that $\left(T_{t}^{-} w\right)(x, \omega)$ is a (sub)solution of (1.16) and that the Hamiltonian is autonomous.

Let us prove item (iii) for any fixed $t>0$. First notice that, by continuity with respect to $s$, the supremum in (2.32) can be taken over a dense and countable subset of $(0,+\infty)$, yielding that $v(t, \cdot, \cdot)$ is a random variable. Moreover, $v(t, \cdot, \omega)$ is the supremum of a family of critical subsolutions whenever $w(\cdot, \omega)$ is a critical subsolution, see Proposition 1.14, i.e. almost surely. This implies that $v(t, \cdot, \omega)$ is a critical subsolution a.s. in $\omega$. The fact that $v(t, \cdot, \omega)$ is almost surely sublinear is obvious from $(i)$ and from the fact that $w \in \mathcal{S}$. It is left to show that $v(t, \cdot, \cdot)$ has stationary increments. Indeed, $w$ has stationary increments, meaning that, for every fixed $z \in \mathbb{R}^{N}$, there exists a random variable $k(\omega)$ and a set $\Omega_{z}$ with probability 1 such that

$$
w(\cdot+z, \omega)=w\left(\cdot, \tau_{z} \omega\right)+k(\omega) \quad \text { on } \mathbb{R}^{N} \text { for every } \omega \in \Omega_{z} .
$$

Keeping in mind the stationary character of the Lagrangian, it is easily checked that the same identity is satisfied by $T_{s}^{-} w$ for every $s>0$, and, consequently, by $v(t, \cdot, \cdot)$.

Assertion (iv) is straightforward consequence of the definition of $v$ in view of Proposition 2.18.

Assertion (v) is also well known, see for instance [5].

Let us prove the last assertion. We first note that, when $w(\cdot, \omega)$ is a critical subsolution, the monotonicity of the map $t \mapsto\left(T_{t}^{-} w\right)(x, \omega)$ readily implies, by the very definition of $v$, that

$$
Y(t, x, \omega) \subset[t,+\infty) .
$$

Assume now that $w$ is weakly strict and let us denote by $\Omega_{w}$ a set of probability 1 made up by elements $\omega$ for which $w(\cdot, \omega)$ is a weakly strict critical subsolution. Fix $\omega \in \Omega_{w}$ and pick a point $x \in \mathbb{R}^{N} \backslash \mathcal{A}(\omega)$. Then we know 
by (the proof of) Proposition 2.19 that there exists $t_{x} \in(0,+\infty]$ such that the function $t \mapsto\left(T_{t}^{-} w\right)(x, \omega)$ is strictly increasing in $\left[0, t_{x}\right)$ and constant for $t \geq t_{x}$. In view of $(2.33)$ and the very definition of $v$, we infer that

$$
t \mapsto v(t, x, \omega) \text { is constant for } t \geq t_{x} .
$$

It is left to show that it is strictly increasing in $\left[0, t_{x}\right)$. Take $t_{1}>t_{2}$ in $\left[0, t_{x}\right)$ and let $s_{i} \in Y\left(t_{i}, x, \omega\right)$ for $i=1,2$. Two cases are possible: either $s_{2}<t_{x}$ or $s_{2}=t_{x}$. In the first instance we have

$$
\begin{aligned}
v\left(t_{2}, x, \omega\right) & =\left(T_{s_{2}}^{-} w\right)(x, \omega)-\frac{1}{2 \delta}\left(s_{2}-t_{2}\right)^{2} \\
& <\left(T_{t_{1}+\left(s_{2}-t_{2}\right)}^{-} w\right)(x, \omega)-\frac{1}{2 \delta}\left(s_{2}-t_{2}\right)^{2} \leq v\left(t_{1}, x, \omega\right),
\end{aligned}
$$

in the second

$$
\begin{aligned}
v\left(t_{2}, x, \omega\right) & =\left(T_{t_{x}}^{-} w\right)(x, \omega)-\frac{1}{2 \delta}\left(t_{x}-t_{2}\right)^{2} \\
& <\left(T_{t_{x}}^{-} w\right)(x, \omega)-\frac{1}{2 \delta}\left(t_{x}-t_{1}\right)^{2} \leq v\left(t_{1}, x, \omega\right),
\end{aligned}
$$

as it was to be shown.

\section{Tonelli Hamiltonians}

In this section we deal with Hamiltonians satisfying more stringent regularity assumptions and named after Tonelli. In the first subsection we provide basic definitions and illustrate the salient features of the corresponding Hamiltonian flow and Lax-Oleinik semigroups. In the second one we prove existence of $C^{1,1}$ strict subsolutions in the stationary ergodic setting and investigate their properties. This is achieved by applying Bernard's method on $\mathrm{C}^{1,1}$-regularization of strict subsolutions on compact manifolds in the deterministic case.

Throughout the section we will use the term semiconcave (respectively semiconvex) in a stronger sense than the one defined in Section 1: we will in fact additionally require the modulus to be linear, namely $\Theta(h)=K h$ for some $K>0$. If such a constant need to be showcased then we will employ the diction $K$-semiconcave (respectively, $K$-semiconvex). We recall that a function $u$ is both $K$-semiconcave and $K$-semiconvex in some open subset $U$ of $\mathbb{R}^{N}$ if and only if is of class $C^{1,1}$ in $U$ and $\operatorname{Lip}(D u ; U) \leq K$, see [5].

\subsection{Generalities}

We say that a stationary ergodic Hamiltonian $H$ is Tonelli if it enjoys conditions (H1)-(H4) and the following set of assumptions:

(T1) $H(\cdot, \cdot, \omega) \in C^{2}\left(\mathbb{R}^{N} \times \mathbb{R}^{N}\right)$ for every $\omega \in \Omega$;

(T2) for every $R>0$ there exists a constant $v_{R}>0$ such that

$$
\frac{\partial_{p}^{2} H}{\partial p^{2}}(x, p, \omega)>v_{R} \operatorname{Id}_{\mathbb{R}^{N}} \quad \text { for every }(x, p, \omega) \in \mathbb{R}^{N} \times B_{R} \times \Omega ;
$$

(T3) for every $R>0$ there exists $M_{R}>0$ such that

$$
\begin{gathered}
\|D H(\cdot, \cdot, \omega)\|_{L^{\infty}\left(\mathbb{R}^{N} \times B_{R}\right)}<M_{R}, \\
\left\|D^{2} H(\cdot, \cdot, \omega)\right\|_{L^{\infty}\left(\mathbb{R}^{N} \times B_{R}\right)}<M_{R}
\end{gathered}
$$

for every $\omega \in \Omega$.

The following holds

Proposition 3.1. The associated Lagrangian L satisfies properties analogous to (T1)-(T3). 
Proof. For every fixed $\omega \in \Omega$, we already know, see for instance [7,16], that $L$ is of class $C^{2}$ in $\mathbb{R}^{N} \times \mathbb{R}^{N}$ and

$$
q=\partial_{p} H\left(x, \partial_{q} L(x, q, \omega), \omega\right), \quad \partial_{x} L(x, q, \omega)=-\partial_{x} H\left(x, \partial_{q} L(x, q, \omega), \omega\right)
$$

for every $(x, q) \in \mathbb{R}^{N} \times \mathbb{R}^{N}$. From assumption (H3) it is easily seen that $L$ is bounded in $\mathbb{R}^{N} \times B_{R}$ for every $R>0$, uniformly with respect to $\omega$. By the convexity of $L$ in $q$, this readily implies that for every $R>0$ there exists $\rho(R)>0$ such that

$$
\left|\partial_{q} L(x, q, \omega)\right| \leq \rho(R) \quad \text { for every }(x, q, \omega) \in \mathbb{R}^{N} \times B_{R} \times \Omega .
$$

Let us fix $R>0$ and let us denote by $M_{\rho(R)}$ a positive constant chosen according to (T3). By differentiating the first equality in (3.1) with respect to $q$ we get

$$
\frac{\partial^{2} L}{\partial q^{2}}(x, q, \omega)=\left(\frac{\partial^{2} H}{\partial p^{2}}\left(x, \partial_{q} L(x, q, \omega), \omega\right)\right)^{-1}>\frac{C}{M_{\rho(R)}} \operatorname{Id}_{\mathbb{R}^{N}}
$$

for every $(x, q, \omega) \in \mathbb{R}^{N} \times B_{R} \times \Omega$ and for some universal constant $C>0$. This proves that $L$ satisfies a condition analogous to (T2). The fact that $D L$ is bounded in $\mathbb{R}^{N} \times B_{R}$ for every $R>0$, uniformly with respect to $\omega$, immediately follows from the second equality in (3.1) and from (3.2). An analogous bound for $D^{2} L$ can be easily proved by differentiating the identities in (3.1) with respect to $x$ and $q$ and by making use of (3.2) and assumption (T3).

Remark 3.2. The above hypotheses, in particular (T2) and (T3), are adaptation to the stationary ergodic environment of the usual ones required for deterministic Tonelli Hamiltonians. The changes are basically due to the fact that we need bounds independent of $\omega$, and this immediately implies that they have also to be global in $x$. In fact, bounds independent of $\omega$ that are local in $x$ simply do not make sense in our frame, for stationarity and ergodicity assumptions should automatically transfer them to the whole $\mathbb{R}^{N}$. Similarly, we could rephrase (T2) and (T3) by requiring $v_{R}$ and $M_{R}$ to depend in a measurable way on $\omega$ : the ergodicity assumption would then imply that they are almost surely constant.

We will denote by $\phi_{t}^{H}(x, p, \omega)$ the Hamiltonian flow, i.e. the flow associated with Hamilton's ODE

$$
\left\{\begin{array}{l}
\dot{\xi}=\partial_{p} H(\xi, \eta, \omega) \\
\dot{\eta}=-\partial_{x} H(\xi, \eta, \omega) .
\end{array}\right.
$$

The corresponding integral curves will be also called characteristics in the sequel. As well known, $H\left(\phi_{t}^{H}(x, p, \omega)\right.$, $\omega)=H(x, p, \omega)$ for every $(x, p, \omega)$, which yields, by the coercivity assumption (H3), that the flow is complete, i.e. globally defined in time.

We proceed discussing the two main additional features of Lax-Oleinik semigroups for Tonelli Hamiltonians. The first one asserts that the action of the negative semigroups is driven by characteristics, see [16] for the proof.

Proposition 3.3. Let $u$ be a $\vartheta$-Lipschitz random function, $x \in \mathbb{R}^{N}$ and $t>0$. Let $\gamma:[0, t] \rightarrow \mathbb{R}^{N}$ be a Lipschitz curve with $\gamma(t)=x$ such that

$$
\left(T_{t}^{-} u\right)(x, \omega)=u(\gamma(0), \omega)+\int_{0}^{t} L(\gamma, \dot{\gamma}, \omega) \mathrm{d} s .
$$

Then $\gamma$ is the projection on the state variable space of a characteristic taking value $\left(\gamma(0), p_{\gamma(0)}\right)$ at time 0 , and $\left(x, p_{x}\right)$ at time $t$, where

$$
p_{\gamma(0)} \in D^{-} u(\gamma(0), \omega), \quad p_{x} \in D^{+}\left(T_{t}^{-} u\right)(x, \omega) .
$$

While for continuous Hamiltonians, we can only assert $\kappa$-Lipschitz continuity of $\left(T_{t}^{-} u\right)(\cdot, \omega)$ and $\left(T_{t}^{+} u\right)(\cdot, \omega)$ when $u \in \mathcal{S}$, for Tonelli ones we get semiconcavity and semiconvexity, respectively. This property will be crucial to transfer to the stationary ergodic setting the regularization procedure yielding $\mathrm{C}^{1,1}$ critical subsolutions. 
Proposition 3.4. Let $t_{0}>0$. Then the functions $\left\{h_{t_{0}}(\cdot, \cdot, \omega): \omega \in \Omega\right\}$ are locally equi-semiconcave (and equiLipschitz) in $\mathbb{R}^{N} \times \mathbb{R}^{N}$. In particular, there exists a constant $K=K\left(t_{0}\right)$ such that, for every $w \in \mathcal{S}$ and for every $t \geq t_{0}$, $\left(T_{t}^{-} w\right)(\cdot, \omega)$ is $K$-semiconcave in $\mathbb{R}^{N},\left(T_{t}^{+} w\right)(\cdot, \omega)$ is $K$-semiconvex in $\mathbb{R}^{N}$, for every $\omega \in \Omega$.

Proof. Let us pick $x_{1}, x_{2} \in \mathbb{R}^{N}$ and $r>0$ and set $U_{i}:=B_{r}\left(z_{i}\right)$ for $i=1,2$. The fact that $h_{t_{0}}(\cdot, \cdot, \omega)$ is, for fixed $\omega$, semiconcave in $U_{1} \times U_{2}$ is well known, see for instance [18, Theorem B.19]. We only need to check that the semiconcavity constant can be chosen independent of $\omega$. To this aim, let us first denote by $\alpha_{L}, \beta_{L}: \mathbb{R}_{+} \rightarrow \mathbb{R}$ two superlinear functions such that

$$
\alpha_{L}(|q|) \leq L(x, q, \omega) \leq \beta_{L}(|q|) \text { for all }(x, q, \omega) \in \mathbb{R}^{N} \times \mathbb{R}^{N} \times \Omega ;
$$

Such functions do exist due to condition (H3). Moreover, they can be assumed, without any loss of generality, convex and nondecreasing, see Remark 1.8. It is not difficult to see that

$$
t_{0} \alpha_{L}\left(\frac{|x-y|}{t_{0}}\right) \leq h_{t_{0}}(y, x, \omega) \leq t_{0} \beta_{L}\left(\frac{|x-y|}{t_{0}}\right) \quad \text { in } \mathbb{R}^{N} \times \mathbb{R}^{N} \times \Omega .
$$

Hence we can always assume that a Lagrangian minimizer $\gamma:\left[0, t_{0}\right] \rightarrow \mathbb{R}^{N}$ with $\gamma(0) \in U_{1}$ and $\gamma\left(t_{0}\right) \in U_{2}$ satisfies $\|\dot{\gamma}\|_{\infty} \leq A$ for some constant $A$ only depending on $r, t_{0}, \alpha_{L}, \beta_{L}$, see for instance Lemma 3.2 in [9]. Let $M$ be a Lipschitz constant for $D L(\cdot, \cdot, \omega)$ in $\mathbb{R}^{N} \times B_{A+4 r / t_{0}}$ for every $\omega \in \Omega$, which exists according to Proposition 3.1. Fix $\omega \in \Omega$ and pick $x_{i}, y_{i} \in U_{i}$ for $i=1,2$ and a Lagrangian minimizer $\gamma:\left[0, t_{0}\right] \rightarrow \mathbb{R}^{N}$ with $\gamma(0)=x_{1}$ and $\gamma\left(t_{0}\right)=x_{2}$ (i.e. an optimal curve for $\left.h_{t_{0}}\left(x_{1}, x_{2}, \omega\right)\right)$ such that $\|\dot{\gamma}\|_{\infty} \leq A$. We define a curve $\tilde{\gamma}:\left[0, t_{0}\right] \rightarrow \mathbb{R}^{N}$ joining $y_{1}$ to $y_{2}$ by setting

$$
\tilde{\gamma}(s)= \begin{cases}\frac{t_{0}-2 s}{t_{0}}\left(y_{1}-x_{1}\right)+\gamma(s) & \text { if } s \in\left[0, t_{0} / 2\right) \\ \frac{2 s-t_{0}}{t_{0}}\left(y_{2}-x_{2}\right)+\gamma(s) & \text { if } s \in\left[t_{0} / 2, t_{0}\right] .\end{cases}
$$

Arguing as in the proof of Theorem B.19 in [18], we derive

$$
\begin{aligned}
h_{t_{0}}\left(y_{1}, y_{2}, \omega\right)-h_{t_{0}}\left(x_{1}, x_{2}, \omega\right) & \leq \int_{0}^{t_{0}} L(\tilde{\gamma}(s), \dot{\tilde{\gamma}}(s), \omega) \mathrm{d} s-\int_{0}^{t_{0}} L(\gamma(s), \dot{\gamma}(s), \omega) \mathrm{d} s \\
& \leq\left\langle p_{x_{1}}(\omega), y_{1}-x_{1}\right\rangle+\left\langle p_{x_{2}}(\omega), y_{2}-x_{2}\right\rangle+M\left(\frac{t_{0}}{2}+\frac{2}{t_{0}}\right)\left(\left|y_{1}-x_{1}\right|^{2}+\left|y_{2}-x_{2}\right|^{2}\right)
\end{aligned}
$$

for a pair of vectors $p_{1}(\omega), p_{2}(\omega) \in \mathbb{R}^{N}$. This proves the first part of the statement.

Let us prove the asserted semiconcavity of $T_{t}^{-} w$. Let us first prove that there exists a constant $K=K\left(t_{0}\right)$ such that $T_{t_{0}} w(\cdot, \omega)$ is $K$-semiconcave in $B_{1}$ for every $\omega \in \Omega$ and $w \in \mathcal{S}$. This is a consequence of the fact that, for every $\omega \in \Omega$,

$$
T_{t_{0}} w(\cdot, \omega)=\min \left\{h_{t_{0}}(y, \cdot, \omega)+w(y, \omega):|y| \leq 1+t_{0} R(\kappa)\right\} \quad \text { in } B_{1}
$$

in view of Proposition 1.12 and that the functions $\left\{h_{t_{0}}(y, \cdot, \omega):|y| \leq 1+t_{0} R(\kappa), \omega \in \Omega\right\}$ are locally equisemiconcave. By the fact that $T_{t_{0}} w$ has stationary increments, it is easily seen that $T_{t_{0}} w(\cdot, \omega)$ is $K$-semiconcave in $B_{1}(z)$ if and only if $T_{t_{0}} w\left(\cdot, \tau_{z} \omega\right)$ is $K$-semiconcave in $B_{1}$. This readily implies that $T_{t_{0}} w(\cdot, \omega)$ is $K$-semiconcave in $\mathbb{R}^{N}$ for every $\omega \in \Omega$. To see that the same semiconcavity constant works for $t>t_{0}$ as well, simply notice that $T_{t} w=T_{t_{0}}\left(T_{t-t_{0}} w\right)$ and $T_{t-t_{0}} w \in \mathcal{S}$ for every $w \in \mathcal{S}$.

The semiconvexity of $T_{t}^{+} w$ readily follows from this in view of Remark 1.11.

We can derive from Theorem 2.12:

Theorem 3.5. The following facts hold:

(i) for every $v \in \mathcal{S}$, there exists a set $\Omega_{v}$ of probability 1 such that for every $\omega \in \Omega_{v}$ and every $x \in \mathcal{A}(\omega)$ $v(\cdot, \omega)$ is differentiable at $x$ and $H(x, D v(x))=0$. 
(ii) Let $u$ and $v$ belong to $\mathcal{S}$. Then

$$
D u(\cdot, \omega)=D v(\cdot, \omega) \quad \text { on } \mathcal{A}(\omega)
$$

for every $\omega \in \Omega_{u} \cap \Omega_{v}$.

Theorem 3.5 is analogous to a well known result in weak KAM Theory and can be proved similarly, see [16].

Let us pick $v \in \mathcal{S}$ and set

$$
\widetilde{\mathcal{A}}(\omega):=\{(x, D v(x, \omega)): x \in \mathcal{A}(\omega)\}, \quad \omega \in \Omega .
$$

Up to a set of null probability, the definition of $\widetilde{\mathcal{A}}(\omega)$ is independent of the choice of $v \in \mathcal{S}$ in view of Theorem 3.5. The following holds, see [16]:

Theorem 3.6. There exists a set $\widehat{\Omega}$ of probability 1 such that for every $\omega \in \widehat{\Omega}$

$$
\phi_{H}^{t}(\widetilde{\mathcal{A}}(\omega), \omega)=\widetilde{\mathcal{A}}(\omega) \quad \text { for every } t \in \mathbb{R} .
$$

3.2. $\mathrm{C}^{1,1}$ subsolutions in stationary ergodic case

In this subsection we show how to pass from a strict critical and admissible subsolution, which is in general just Lipschitz-continuous with respect to $x$, to one which is of class $C^{1,1}$ in $\mathbb{R}^{N}$. The precise result we will prove is the following:

Theorem 3.7. Let H satisfy (H1)-(H4) and (T1)-(T3). Then there exists a strict critical subsolution in $\mathcal{S}$ of class $C^{1,1}$ in $\mathbb{R}^{N}$. More precisely, for every strict critical subsolution $w \in \mathcal{S}$ and every $\varepsilon>0$, there exists a strict critical subsolution $w_{\varepsilon} \in \mathcal{S}$ such that

(i) $\left\|w_{\varepsilon}(\cdot, \omega)-w(\cdot, \omega)\right\|_{\infty}<\varepsilon$ for every $\omega \in \Omega$;

(ii) $w_{\varepsilon}(\cdot, \omega)=w(\cdot, \omega)$ on $\mathcal{A}(\omega)$ a.s. in $\omega$;

(iii) $w_{\varepsilon}(\cdot, \omega) \in \mathrm{C}^{1,1}\left(\mathbb{R}^{N}\right)$ for every $\omega \in \Omega$.

This theorem, in particular, implies the existence of a critical admissible subsolution of class $\mathrm{C}^{1,1}$ and strict on the whole $\mathbb{R}^{N}$ when the random Aubry set is almost surely empty.

As a direct consequence of Theorem 3.7 and Corollary 2.17 we get

Corollary 3.8. Let $H$ satisfy (H1)-(H4) and (T1)-(T3). Then the set of admissible, strict critical subsolutions of class $C^{1,1}$ in $\mathbb{R}^{N}$ is dense in $\mathcal{S}$ with respect to the Ky Fan metric on $L^{0}\left(\Omega ; \mathrm{C}\left(\mathbb{R}^{N}\right)\right)$.

We start by showing a further invariance property of Lax-Oleinik semigroup.

Proposition 3.9. Let $H$ be a stationary ergodic Tonelli Hamiltonian. Then the family of strict subsolution belonging to $\mathcal{S}$ is invariant for the positive and negative Lax-Oleinik semigroups. Namely, if $u \in \mathcal{S}$ and is strict, then both $T_{t}^{+} u$ and $T_{t}^{-} u$ belong to $\mathcal{S}$ and are strict, for every $t>0$.

Proof. Let $u \in \mathcal{S}$ be strict. Take $\omega$ such that $u(\cdot, \omega)$ is a strict critical subsolution, $u(\cdot, \omega)$ is differentiable on $\mathcal{A}(\omega)$ and $\widetilde{\mathcal{A}}(\omega)$ is invariant with respect to the Hamiltonian flow. Theorems 3.5 and 3.6 guarantee that such a choice can be done in set of probability 1 . We go on fixing $t>0$ and a closed ball $B$ disjoint from $\mathcal{A}(\omega)$. We consider the set $V$ made up by points $y$ optimal for $\left(T_{t}^{-} u\right)(x, \omega)$, for some $x \in B$. This set inherits from $B$ the property of being compact. As established in Proposition 3.3, if $y \in V$ is optimal for $\left(T_{t}^{-} u\right)(x, \omega)$, the corresponding Lagrangian minimizer $\gamma:[0, t] \rightarrow \mathbb{R}^{N}$ is the projection on the state variable space of the characteristic taking the value $(y, p)$ at time 0 , with $p \in D^{-} u(y, \omega)$. If $y \in \mathcal{A}(\omega)$, then $p=D u(y, \omega)$ and $(y, p) \in \widetilde{\mathcal{A}}(\omega)$, but then, by the invariance property of $\widetilde{\mathcal{A}}(\omega), \gamma$ cannot reach $x$, which does not belong to $\mathcal{A}(\omega)$. This argument shows by contradiction that $V \cap \mathcal{A}(\omega)=\emptyset$. 
Being $u(\cdot, \omega)$ strict critical subsolution, there exists $\delta>0$ with

$$
H(y, p, \omega) \leq-\delta \quad \text { for every } p \in \partial^{c} u(y, \omega) \text { and } y \in V .
$$

Taking into account Proposition 3.3 and the fact that the Hamiltonian stays constant on characteristics, we derive

$$
H\left(x, D_{x}\left(T_{t}^{-} u\right)(x, \omega), \omega\right) \leq-\delta \quad \text { for a.e. } x \in B .
$$

Since $B$ is a closed ball arbitrarily chosen in the complement of $\mathcal{A}(\omega)$, this proves the assertion for $T_{t}^{-} u$, and consequently for $T_{t}^{+} u$ in view of Remark 1.11 .

The above argument shows that, if $x \notin \mathcal{A}(\omega)$, the optimal points for $T_{t}^{-} u(x, \omega)$ do not belong to $\mathcal{A}(\omega)$ for any $t>0$. Thanks to this, we get the following strengthened form of Proposition 2.19 for Tonelli Hamiltonians:

Corollary 3.10. Let $H$ be a stationary ergodic Tonelli Hamiltonian. Then, for any weakly strict subsolution $w \in \mathcal{S}$, there exists a set $\Omega_{w}$ of probability 1 such that, for every $\omega \in \Omega_{w}$ and $x \in \mathbb{R}^{N} \backslash \mathcal{A}(\omega)$, the function

$$
t \mapsto\left(T_{t}^{-} w\right)(x, \omega)
$$

is strictly increasing in $[0,+\infty)$. In particular, $T_{t}^{-} w$ is weakly strict for every $t>0$.

For the sake of completeness, we also provide, in the framework of the proof of Theorem 3.7, a worked out presentation of Bernard regularization technique in the deterministic setting using our terminology and notations. The material is illustrated in a more elementary way and providing more details with respect to the original proof in [3], at the expense of some loss of concision and elegance.

The first two steps are purely deterministic, and so $\omega$ is omitted. Lemma 3.11 establish that a function which is $C^{1,1}$ locally around a given point, remains of class $C^{1,1}$, at least in a smaller neighborhood of the same point, under application of $T_{t}^{-}$for small times. It will be used in the subsequent Proposition 3.12 to show, by working on subtangents, that a function locally semiconvex becomes locally $\mathrm{C}^{1,1}$ under the same action.

In what follows, the symbols $\pi_{1}$ and $\pi_{2}$ will denote the projections on the space and momentum variable, respectively, i.e. the maps defined as $\pi_{1}(x, p)=x, \pi_{2}(x, p)=p$ for every $(x, p) \in \mathbb{R}^{N} \times \mathbb{R}^{N}$.

Lemma 3.11. Assume $\psi: \mathbb{R}^{N} \rightarrow \mathbb{R}$ to be $\widetilde{\kappa}$-Lipschitz-continuous in $\mathbb{R}^{N}$, for some $\widetilde{\kappa}>0$ and, in addition, of class $C^{1,1}$ in a neighborhood of $B_{1}\left(x_{0}\right)$, for some $x_{0} \in \mathbb{R}^{N}$. Then there exist $t_{0}>0$ and $A>0$, solely depending on $\widetilde{\kappa}$ and the Lipschitz constant of $D \psi$ in $B_{1}\left(x_{0}\right)$, such that, for every $t \in\left[0, t_{0}\right]$, the following properties hold:

(i) any $y \in B_{1 / 2}\left(x_{0}\right)$ is the unique optimal point in $\mathbb{R}^{N}$ for $\left(T_{t}^{-} \psi\right)(x)$ with $x=\pi_{1} \circ \phi_{t}^{H}(y, D(\psi(y)))$;

(ii) $T_{t}^{-} \psi \in \mathrm{C}^{1,1}\left(B_{1 / 2}\left(x_{0}\right)\right)$ and $\operatorname{Lip}\left(D_{x}\left(T_{t}^{-} \psi\right) ; B_{1 / 2}\left(x_{0}\right)\right) \leq A$.

Proof. For every $t \geq 0$, we define a map $R_{t}: B_{1}\left(x_{0}\right) \rightarrow \mathbb{R}^{N}$ by setting

$$
R_{t}(y)=\pi_{1} \circ \phi_{t}^{H}(y, D \psi(y)) \text { for every } y \in B_{1}\left(x_{0}\right) .
$$

We claim that we can choose $t_{0}>0$, only depending on $\widetilde{\kappa}$ and on the Lipschitz constant of $D \psi$ in $B_{1}\left(x_{0}\right)$, such that the map $R_{t}-I$ is a contraction on $B_{1}\left(x_{0}\right)$ for every $t \in\left[0, t_{0}\right]$. To this aim, fix $y_{1}$ and $y_{2}$ in $B_{1}\left(x_{0}\right)$ and denote by $\left(\xi_{i}, \eta_{i}\right)$, $i=1,2$, the characteristic taking the value $\left(y_{i}, D \psi\left(y_{i}\right)\right)$ at 0 . We have

$$
R_{t}\left(y_{i}\right)-y_{i}=\int_{0}^{t} \partial_{p} H\left(\xi_{i}, \eta_{i}\right) \mathrm{d} s
$$

and consequently

$$
\left|\left(R_{t}\left(y_{1}\right)-y_{1}\right)-\left(R_{t}\left(y_{2}\right)-y_{2}\right)\right| \leq \int_{0}^{t}\left|\partial_{p} H\left(\xi_{1}, \eta_{1}\right)-\partial_{p} H\left(\xi_{2}, \eta_{2}\right)\right| \mathrm{d} s .
$$


Now we recall that $H$ is superlinear and locally bounded in $p$, uniformly in $x$, and stays constant on characteristics. From the inequality $\left|D \psi\left(y_{i}\right)\right| \leq \widetilde{\kappa}$ we derive that $\left(\xi_{i}(s), \eta_{i}(s)\right)$ is contained $\mathbb{R}^{N} \times B_{\rho}$ for some $\rho$ only depending on $\widetilde{\kappa}$ and on the functions $\alpha_{H}, \beta_{H}$ appearing in assumption (H3). Let us denote by $M$ the positive constant $M_{R}$ given by hypothesis (T3) with $R=\rho$. Resuming our estimate, from (3.3) we get

$$
\left|\left(R_{t}\left(y_{1}\right)-y_{1}\right)-\left(R_{t}\left(y_{2}\right)-y_{2}\right)\right| \leq M \int_{0}^{t}\left|\left(\xi_{1}, \eta_{1}\right)-\left(\xi_{2}, \eta_{2}\right)\right| \mathrm{d} s .
$$

Let us denote by $\lambda$ a Lipschitz constant for $D \psi$ in $B_{1}\left(x_{0}\right)$. By Gronwall inequality

$$
\begin{aligned}
\left|\left(\xi_{1}(s), \eta_{1}(s)\right)-\left(\xi_{2}(s), \eta_{2}(s)\right)\right| & \leq e^{M s}\left|\left(y_{1}, D \psi\left(y_{1}\right)\right)-\left(y_{2}, D \psi\left(y_{2}\right)\right)\right| \\
& \leq e^{M s}\left(\sqrt{1+\lambda^{2}}\right)\left|y_{1}-y_{2}\right| \quad \text { for } s \in[0, t],
\end{aligned}
$$

hence

$$
\left|\left(R_{t}\left(y_{1}\right)-y_{1}\right)-\left(R_{t}\left(y_{2}\right)-y_{2}\right)\right| \leq\left(\sqrt{1+\lambda^{2}}\right)\left(e^{M t}-1\right)\left|y_{1}-y_{2}\right| .
$$

Let us choose $t_{0}>0$ such that

$$
\left(e^{M t_{0}}-1\right) \sqrt{1+\lambda^{2}}<\frac{1}{2}, \quad M t_{0}<\frac{1}{4}, \quad t_{0} R(\widetilde{\kappa})<\frac{1}{4},
$$

with $R(\widetilde{\kappa})$ given by Proposition 1.12, and fix $t \in\left[0, t_{0}\right]$. From (3.5) we derive that $R_{t}-I$ is a contraction, with Lipschitz constant less than $1 / 2$. By the triangular inequality we get

$$
\frac{1}{2}\left|y_{1}-y_{2}\right| \leq\left|R_{t}\left(y_{1}\right)-R_{t}\left(y_{2}\right)\right| \leq \frac{3}{2}\left|y_{1}-y_{2}\right| \text { for every } y_{1}, y_{2} \in B_{1}\left(x_{0}\right),
$$

showing in particular that $R_{t}$ is injective in $B_{1}\left(x_{0}\right)$.

Let us prove $(i)$. We first observe that the equality

$$
\left(T_{t}^{-} \psi\right)(x)=\psi(y)+h_{t}(y, x) \quad \text { for } x \in B_{3 / 4}\left(x_{0}\right)
$$

implies $y \in B_{1}\left(x_{0}\right)$ and $R_{t}(y)=x$, in view of Proposition 1.12, see the third inequality in (3.6), and of Proposition 3.3, in particular such a point $y$ is unique by injectivity of $R_{t}$. On the other hand, by the choice of $M$ and the inequality $M t<1 / 4$, for every $y \in B_{1 / 2}\left(x_{0}\right)$ we have that the point $x=R_{t}(y)$ belongs to $B_{3 / 4}\left(x_{0}\right)$. In view of the previous remark, we conclude that $y$ is the unique point in $\mathbb{R}^{N}$ that is optimal for $\left(T_{t}^{-} \psi\right)(x)$.

Let us prove (ii). The previous argument implies that $B_{3 / 4}\left(x_{0}\right) \subseteq R_{t}\left(B_{1}\left(x_{0}\right)\right)$, in particular we infer that the map

$$
R_{t}^{-1}: B_{1 / 2}\left(x_{0}\right) \rightarrow B_{1}\left(x_{0}\right)
$$

is well defined and Lipschitz-continuous. From this and (3.4) we get that the function

$$
x \mapsto \pi_{2} \circ \phi_{t}^{H}\left(R_{t}^{-1}(x), D\left(\psi\left(R_{t}^{-1}(x)\right)\right)\right)
$$

is $A$-Lipschitz continuous in $B_{1 / 2}\left(x_{0}\right)$, where $A$ is a constant only depending on $t_{0}$ and on the Lipschitz constant of $D \psi$ in $B_{1}\left(x_{0}\right)$. In addition, by Proposition 3.3, its images belong, for any $x$, to $D^{+}\left(T_{t}^{-} \psi\right)(x)$. This means that we have constructed an $A$-Lipschitz continuous selection of $D^{+}\left(T_{t}^{-} \psi\right)(x)$ in $B_{1 / 2}\left(x_{0}\right)$. Since $D^{+}\left(T_{t}^{-} \psi\right)(x)$ reduces to the differential of $T_{t}^{-} \psi$ at any differentiability point, we infer, by its very definition, that the Clarke generalized gradient of $T_{t}^{-} \psi$ reduces to a singleton at any point of $B_{1 / 2}\left(x_{0}\right)$. This readily gives that $T_{t}^{-} \psi \in \mathrm{C}^{1,1}\left(B_{1 / 2}\left(x_{0}\right)\right)$ and $\operatorname{Lip}\left(D_{x}\left(T_{t}^{-} \psi\right) ; B_{1 / 2}\left(x_{0}\right)\right) \leq A$.

Proposition 3.12. Let $w$ be a critical subsolution which is $K$-semiconvex in $B_{1}\left(x_{0}\right)$ for some $x_{0} \in \mathbb{R}^{N}$. Then we can find $t_{0}>0$, only depending on $K$, such that

$$
T_{t}^{-} w \in \mathrm{C}^{1,1}\left(B_{1 / 4}\left(x_{0}\right)\right) \quad \text { for any } t \in\left(0, t_{0}\right] .
$$

Moreover, $\operatorname{Lip}\left(D_{x}\left(T_{t}^{-} w\right) ; B_{1 / 4}\left(x_{0}\right)\right) \leq B_{t}$ for some $B_{t}$ only depending on $t$ and $K$. 
Proof. We recall that, being $w$ a critical subsolution, it is $\kappa$-Lipschitz continuous, with $\kappa$ given by (1.8) with $a=0$, i.e. the critical value. By semiconvexity assumption on $w$ we have that, for any $y \in B_{1}\left(x_{0}\right)$ and $p \in D^{-} w(y)$, the paraboloid

$$
\psi_{y, p}(x)=w(y)+\langle p, x-y\rangle-K|x-y|^{2}, \quad x \in B_{1}\left(x_{0}\right)
$$

satisfies $\psi_{y, p} \leq w$ in $B_{1}\left(x_{0}\right)$. We suitably extend it outside $B_{1}\left(x_{0}\right)$ to obtain a family of $\widetilde{\kappa}$-Lipschitz continuous subtangents to $w$ in $\mathbb{R}^{N}$, i.e. satisfying $\psi \leq w$ in $\mathbb{R}^{N}$, which clearly are smooth and have $K$-Lipschitz differentials in $B_{1}\left(x_{0}\right)$. Here $\widetilde{\kappa}$ is a constant greater than $\kappa$. We denote by $\mathcal{F}$ this family of modified paraboloids.

In view of Lemma 3.11, there exist two common values $t_{0}>0$ and $A>0$ such that, for every $t \in\left[0, t_{0}\right]$, all functions of $\mathcal{F}$ satisfy items $(i)$ and $(i i)$ of the lemma. We recall that $t_{0}$ has been chosen small enough so that

$$
t_{0} R(\widetilde{\kappa})<\frac{1}{4},
$$

where $R(\widetilde{\kappa})$ is the constant given by Proposition 1.12. Let us fix $t \in\left[0, t_{0}\right]$. We want to show that

$$
\left(T_{t}^{-} w\right)(x)=\sup _{\psi \in \mathcal{F}}\left(T_{t}^{-} \psi\right)(x) \quad \text { for every } x \in B_{1 / 4}\left(x_{0}\right)
$$

By monotonicity of $T_{t}^{-}$, it is clear that $T_{t}^{-} w \geq \sup _{\psi \in \mathcal{F}} T_{t}^{-} \psi$ on $\mathbb{R}^{N}$. Let us show equality on $B_{1 / 4}\left(x_{0}\right)$. Fix $x \in$ $B_{1 / 4}\left(x_{0}\right)$ and let $y \in \mathbb{R}^{N}$ be optimal for $\left(T_{t}^{-} w\right)(x)$. Then $y \in B_{1 / 2}\left(x_{0}\right)$ in view of Proposition 1.12 and of (3.7). By Proposition 3.3, there furthermore exists $p \in D^{-} w(y)$ such that

$$
x=\pi_{1} \circ \phi_{t}^{H}(y, p) .
$$

By the very definition of $\mathcal{F}$, there is $\psi \in \mathcal{F}$ which is a subtangent to $w$ at $y$ and satisfies $D \psi(y)=p$. Hence, by Lemma 3.11-(i), $y$ is also optimal for $\left(T_{t}^{-} \psi\right)(x)$, so that

$$
\left(T_{t}^{-} w\right)(x)=\left(T_{t}^{-} \psi\right)(x),
$$

proving (3.8). Summing up, we have showed that $T_{t}^{-} w$ is the supremum in $B_{1 / 4}\left(x_{0}\right)$ of a family of $A$-semiconvex functions, so it is $A$-semiconvex. Now $T_{t}^{-} w$ is also $K_{t}$-semiconcave for some $K_{t}$ only depending on $t$, in view of Proposition 3.4. We conclude that $T_{t}^{-} w$ is of class $C^{1,1}$ in $B_{1 / 4}\left(x_{0}\right)$ with $\operatorname{Lip}\left(D_{x}\left(T_{t}^{-} w\right) ; B_{1 / 4}\left(x_{0}\right)\right) \leq B_{t}:=\max \left\{A, K_{t}\right\}$.

We go back to stationary ergodic setting and apply the information on the action of Lax-Oleinik semigroups gathered so far.

Proof of Theorem 3.7. Let $w \in \mathcal{S}$ be a strict subsolution and let $\varepsilon>0$ be fixed. Choose $s>0$ such that $R(\kappa) s<\varepsilon / 2$ and set

$$
v(x, \omega)=\left(T_{s}^{+} w\right)(x, \omega), \quad(x, \omega) \in \mathbb{R}^{N} \times \Omega .
$$

According to Proposition 3.9, $v$ is still an admissible and strict critical subsolution. Furthermore, by Proposition 3.4, there exists $K>0$ such that $v(\cdot, \omega)$ is $K$-semiconvex in $\mathbb{R}^{N}$ for every $\omega \in \Omega$. In view of Proposition 3.12, there exist $t>0$ and a constant $K_{t}$, only depending on $t$, such that $T_{t}^{-} v(\cdot, \omega)$ is a function of class $C^{1,1}$ in $\mathbb{R}^{N}$ and $\operatorname{Lip}\left(D_{x}\left(T_{t}^{-} v(\cdot, \omega)\right) ; \mathbb{R}^{N}\right) \leq K_{t}$, for every $\omega \in \Omega$. Since $t$ can be taken arbitrarily small, we choose $t>0$ such that $R(\kappa) t<\varepsilon / 2$ and we set

$$
w_{\varepsilon}(x, \omega)=\left(T_{t}^{-} v\right)(x, \omega)=\left(T_{t}^{-} \circ T_{s}^{+} w\right)(x, \omega), \quad(x, \omega) \in \mathbb{R}^{N} \times \Omega .
$$

According to Proposition 3.9, $w_{\varepsilon}$ is still an admissible and strict critical subsolution. From Proposition 1.12 we get

$$
\begin{aligned}
\left\|w_{\varepsilon}(\cdot, \omega)-w(\cdot, \omega)\right\|_{\infty} & \leq\left\|T_{t}^{-} \circ T_{s}^{+} w(\cdot, \omega)-T_{s}^{+} w(\cdot, \omega)\right\|_{\infty}+\left\|T_{s}^{+} w(\cdot, \omega)-w(\cdot, \omega)\right\|_{\infty} \\
& \leq(t+s) R(\kappa)<\varepsilon .
\end{aligned}
$$

Last, by Proposition 2.18, for every $\omega$ in a set of probability 1 we have

$$
w_{\varepsilon}(\cdot, \omega)=T_{t}^{-} \circ T_{s}^{+} w(\cdot, \omega)=T_{s}^{+} w(\cdot, \omega)=w(\cdot, \omega) \quad \text { on } \mathcal{A}(\omega) .
$$




\section{Conflict of interest statement}

We wish to confirm that there are no known conflicts of interest associated with this publication and there has been no financial support for this work that could have influenced its outcome.

\section{Appendix A}

In this appendix, we want to prove a comparison principle between Lipschitz continuous sub and supersolutions of

$$
\partial_{t} u+F\left(x, D_{x} u\right)=0 \quad \text { in }(0,+\infty) \times \mathbb{R}^{N},
$$

where the Hamiltonian $F: \mathbb{R}^{N} \times \mathbb{R}^{N} \rightarrow \mathbb{R}$ is a continuous function satisfying

(F) for every $R>0$ there exists a constant $C_{R}>0$ such that

$$
\left|F\left(x, p_{1}\right)-F\left(x, p_{2}\right)\right| \leq C_{R}\left|p_{1}-p_{2}\right| \quad \text { for every } x \in \mathbb{R}^{N} \text { and } p_{1}, p_{2} \in B_{R} .
$$

We will prove the following result:

Theorem A.1. Let $F: \mathbb{R}^{n} \times \mathbb{R}^{n} \rightarrow \mathbb{R}$ be a Hamiltonian satisfying $(F)$ and let $v, u \in \operatorname{Lip}\left([0, \infty) \times \mathbb{R}^{N}\right)$ be a sub and a supersolution of (A.1), respectively. Then

$$
v(t, x)-u(t, x) \leq \sup _{y \in \mathbb{R}^{N}} v(0, y)-u(0, y) \quad \text { for every }(t, x) \in(0,+\infty) \times \mathbb{R}^{N} .
$$

\section{Proof. Choose}

$$
R>\left\|D_{x} u\right\|_{L^{\infty}\left(\mathbb{R}^{N}\right)}+\left\|D_{x} v\right\|_{L^{\infty}\left(\mathbb{R}^{N}\right)}
$$

and set $C:=C_{R}$ according to hypothesis $(\mathrm{F})$. We claim that the function $w(t, x):=(v-u)(t, x)$ is a viscosity subsolution of

$$
\partial_{t} w-C\left|D_{x} w\right|=0 \quad \text { in }(0,+\infty) \times \mathbb{R}^{N} .
$$

This is enough to conclude since any constant function is a solution of (A.2) and this latter equation satisfies a comparison principle, see for instance [1] or the Appendix in [15]. Let us prove that $w$ is a subsolution of (A.2). Formally the result is obvious since it suffices to subtract the inequalities for $v$ and $u$ and use (F), but to show it in the viscosity sense is a bit more technical. Let $\varphi$ be a $C^{1}$ test function such that $w-\varphi$ attains a strict local maximum at a point $\left(t_{0}, x_{0}\right) \in(0,+\infty) \times \mathbb{R}^{N}$. Let $U$ be an open ball centered at $\left(t_{0}, x_{0}\right)$ and contained in $(0,+\infty) \times \mathbb{R}^{N}$ such that $\left(t_{0}, x_{0}\right)$ is a strict maximum point of $w-\varphi$ in $U$. For $\varepsilon>0$, we introduce the function

$$
\Phi(t, x, s, y):=\frac{1}{2 \varepsilon}\left(|x-y|^{2}+|t-s|^{2}\right)+\varphi(t, x)
$$

and denote by $\left(t_{\varepsilon}, x_{\varepsilon}, s_{\varepsilon}, y_{\varepsilon}\right)$ a maximum point of $v(t, x)-u(s, y)-\Phi(t, x, s, y)$ in $\bar{U} \times \bar{U}$. It is easily shown that both $\left(t_{\varepsilon}, x_{\varepsilon}\right)$ and $\left(s_{\varepsilon}, y_{\varepsilon}\right)$ converge to $\left(t_{0}, x_{0}\right)$ as $\varepsilon \rightarrow 0^{+}$, in particular they lie in $U$ for $\varepsilon>0$ small enough. Hence $\chi(t, x):=\Phi\left(t, x, s_{\varepsilon}, y_{\varepsilon}\right)$ is a supertangent to $v$ at $\left(t_{\varepsilon}, x_{\varepsilon}\right)$ and $\psi(s, y):=-\Phi\left(t_{\varepsilon}, x_{\varepsilon}, s, y\right)$ is a subtangent to $u$ at $\left(s_{\varepsilon}, y_{\varepsilon}\right)$. Since $v$ is a subsolution and $u$ is a supersolution of (A.1), we get

$$
\partial_{t} \chi\left(t_{\varepsilon}, x_{\varepsilon}\right)-\partial_{s} \psi\left(s_{\varepsilon}, y_{\varepsilon}\right) \leqslant F\left(y_{\varepsilon}, D_{x} \psi\left(s_{\varepsilon}, y_{\varepsilon}\right)\right)-F\left(x_{\varepsilon}, D_{x} \chi\left(t_{\varepsilon}, x_{\varepsilon}\right)\right)
$$

The fact that $v\left(t_{\varepsilon}, \cdot\right)$ and $u\left(s_{\varepsilon}, \cdot\right)$ are $R$-Lipschitz and $\chi$ and $\psi$ are a super and a subtangent to $v$ and $u$, respectively, yields

$$
\left|D_{x} \chi\left(t_{\varepsilon}, x_{\varepsilon}\right)\right| \leq R, \quad\left|D_{x} \psi\left(s_{\varepsilon}, y_{\varepsilon}\right)\right| \leq R,
$$

while a direct computation shows that

$$
\partial_{t} \chi\left(t_{\varepsilon}, x_{\varepsilon}\right)-\partial_{s} \psi\left(s_{\varepsilon}, y_{\varepsilon}\right)=\partial_{t} \varphi\left(t_{\varepsilon}, x_{\varepsilon}\right), \quad D_{x} \chi\left(t_{\varepsilon}, x_{\varepsilon}\right)=D_{x} \varphi\left(t_{\varepsilon}, x_{\varepsilon}\right)+D_{x} \psi\left(s_{\varepsilon}, y_{\varepsilon}\right) .
$$


By condition (F) and the choice of $C$ we infer from (A.3)

$$
\partial_{t} \varphi\left(t_{\varepsilon}, x_{\varepsilon}\right) \leq C\left|D_{x} \varphi\left(t_{\varepsilon}, x_{\varepsilon}\right)\right|+\max _{|p| \leq R}\left|F\left(x_{\varepsilon}, p\right)-F\left(y_{\varepsilon}, p\right)\right|,
$$

so the claim follows from the continuity of $F$ by sending $\varepsilon \rightarrow 0^{+}$.

\section{References}

[1] M. Bardi, I. Capuzzo Dolcetta, Optimal Control and Viscosity Solutions of Hamilton-Jacobi-Bellman Equations, with appendices by Maurizio Falcone and Pierpaolo Soravia, Systems \& Control: Foundations \& Applications, Birkhäuser Boston, Inc., Boston, MA, 1997.

[2] G. Barles, Solutions de viscositè des équations de Hamilton-Jacobi, Math. Appl., vol. 17, Springer-Verlag, Paris, 1994.

[3] P. Bernard, Existence of $C^{1,1}$ subsolution of the Hamilton-Jacobi equation on compact manifolds, Ann. Sci. Éc. Norm. Super. 40 (2007) 445-452.

[4] G. Buttazzo, M. Giaquinta, S. Hildebrandt, One-dimensional Variational Problems. An Introduction, Oxf. Lect. Ser. Math. Appl., vol. 15, The Clarendon Press, Oxford University Press, New York, 1998.

[5] P. Cannarsa, C. Sinestrari, Semiconcave Functions, Hamilton-Jacobi Equations, and Optimal Control, Prog. Nonlinear Differ. Equ. Appl., vol. 58, Birkhäuser Boston, Inc., Boston, MA, 2004.

[6] F.H. Clarke, Optimization and Nonsmooth Analysis, second edition, Class. Appl. Math., vol. 5, Society for Industrial and Applied Mathematics (SIAM), Philadelphia, PA, 1990.

[7] G. Contreras, R. Iturriaga, Global minimizers of autonomous lagrangians, in: 22nd Brazilian Mathematics Colloquium, IMPA, Rio de Janeiro, 1999.

[8] G. Dal Maso, H. Frankowska, Value functions for Bolza problems with discontinuous Lagrangians and Hamilton-Jacobi inequalities, ESAIM Control Optim. Calc. Var. 5 (2000) 369-393.

[9] A. Davini, Bolza Problems with discontinuous Lagrangians and Lipschitz continuity of the value function, SIAM J. Control Optim. 46 (5) (2007) 1897-1921.

[10] A. Davini, A. Siconolfi, Weak KAM Theory topics in the stationary ergodic setting, Calc. Var. Partial Differ. Equ. 44 (3-4) (2012) 319-350.

[11] A. Davini, A. Siconolfi, Metric techniques for convex stationary ergodic Hamiltonians, Calc. Var. Partial Differ. Equ. 40 (2011) $391-421$.

[12] A. Davini, A. Siconolfi, Exact and approximate correctors for stochastic Hamiltonians: the 1-dimensional case, Math. Ann. 345 (4) (2009) 749-782.

[13] A. Davini, A. Siconolfi, A generalized dynamical approach to the large time behavior of solutions of Hamilton-Jacobi equations, SIAM J. Math. Anal. 38 (2) (2006) 478-502.

[14] A. Davini, M. Zavidovique, Weak KAM theoretic aspects for nonregular commuting Hamiltonians, Discrete Contin. Dyn. Syst., Ser. B 18 (2013) 1.

[15] A. Davini, M. Zavidovique, On the (non) existence of viscosity solutions of multi-time Hamilton-Jacobi equations, J. Differ. Equ. (2014), http://dx.doi.org/10.1016/j.jde.2014.09.015, in press.

[16] A. Fathi, Weak Kam Theorem in Lagrangian Dynamics. Ghost Book, 2010.

[17] A. Fathi, Weak KAM from a PDE point of view: viscosity solutions of the Hamilton-Jacobi equation and Aubry set, Proc. R. Soc. Edinb. A 142 (6) (2012) 1193-1236.

[18] A. Fathi, A. Figalli, Optimal transportation on non-compact manifolds, Isr. J. Math. 175 (2010) 1-59.

[19] A. Fathi, A. Figalli, L. Rifford, On the Hausdorff dimension of the Mather quotient, Commun. Pure Appl. Math. 62 (4) (2009) 445-500.

[20] A. Fathi, A. Siconolfi, Existence of $C^{1}$ critical subsolutions of the Hamilton-Jacobi equation, Invent. Math. 155 (2004) $363-388$.

[21] A. Fathi, A. Siconolfi, PDE aspects of Aubry-Mather theory for continuous convex Hamiltonians, Calc. Var. Partial Differ. Equ. 22 (2) (2005) $185-228$.

[22] V.V. Jikov, S.M. Kozlov, O.A. Oleinik, Homogenization of Differential Operators and Integral Functionals, translated from the Russian by G.A. Yosifian, Springer-Verlag, Berlin, 1994.

[23] P.L. Lions, P.E. Souganidis, Correctors for the homogenization of Hamilton-Jacobi equations in the stationary ergodic setting, Commun. Pure Appl. Math. 56 (10) (2003) 1501-1524.

[24] I. Molchanov, Theory of Random Sets, Probab. Appl. (New York), Springer-Verlag London, Ltd., London, 2005.

[25] F. Rezakhanlou, J.E. Tarver, Homogenization for stochastic Hamilton-Jacobi equations, Arch. Ration. Mech. Anal. 151 (4) (2000) $277-309$.

[26] P.E. Souganidis, Stochastic homogenization of Hamilton-Jacobi equations and some applications, Asymptot. Anal. 20 (1) (1999) 1-11.

[27] B. Tsirelson, Filtrations of random processes in the light of classification theory. I. A topological zero-one law, preprint, arXiv:math/0107121, 2001. 